\title{
Stahel-Donoho Estimators with Cellwise Weights
}

\author{
S. Van Aelst ${ }^{\mathrm{a}, *}$, E. Vandervieren ${ }^{\mathrm{b}}$, G. Willems ${ }^{\mathrm{a}}$ \\ ${ }^{a}$ Department of Applied Mathematics and Computer Science, Ghent University, \\ Krijgslaan 281 S9, B-9000 Ghent, Belgium \\ ${ }^{\mathrm{b}}$ Department of Mathematics $\&$ Computer Science, University of Antwerp, \\ Middelheimlaan 1, B-2020 Antwerp, Belgium
}

\begin{abstract}
The Stahel-Donoho estimator is defined as a weighted mean and covariance, where each observation receives a weight in function of a measure of its outlyingness. Therefore, all variables are treated in the same way whether they are responsible for the outlyingness or not. We present an adaptation of the Stahel-Donoho estimator where we allow separate weights for each variable. By using cellwise weights, we aim to only downweight the contaminated variables such that we avoid losing the information contained in the other variables. The goal is to increase the precision and possibly the robustness of the estimator. We compare several variants of our proposal and show to what extent they succeed in identifying and downweighting precisely those variables which are contaminated. We further demonstrate that the mean squared error of the adapted estimators is lower than that of the original StahelDonoho estimator in many situations. We also consider some real data examples.
\end{abstract}

Key words: robust multivariate estimators, contamination models, outlier identification.

\section{Introduction}

The Stahel-Donoho estimator, proposed independently by Stahel (1981) and Donoho (1982), is a well-known robust estimator of multivariate location and scatter. It was the first affine equivariant estimator with breakdown point (i.e., the maximum proportion of outliers that the estimator can withstand) close to

* Corresponding author. Tel: +32-9-2644908. Fax:+32-9-2644995

Email address: Stefan.VanAelst@UGent.be 
$50 \%$ for any dimension. It has some excellent robustness properties as shown by Maronna and Yohai (1995), Gervini (2002) and Zuo et al. (2004), and is in this respect comparable to other popular high-breakdown estimators such as the minimum covariance determinant (MCD) estimator (Rousseeuw 1984) or S-estimators (Davies 1987).

The Stahel-Donoho estimator is defined as a weighted mean and covariance, where each observation receives a weight in function of a measure of its "outlyingness". This measure is based on the one-dimensional projection in which the observation is most outlying, the underlying idea of which is that every multivariate outlier must be a univariate outlier in some projection. Hence, observations with large outlyingness then receive small weights. Recent applications of the Stahel-Donoho outlyingness measure can be found in Hubert et al. (2005), Hubert and Verboven (2003) and Debruyne and Hubert (2008).

Whether a large outlyingness is due to an aberrant value in one or more specific variables (componentwise outliers) or to a deviating covariance structure involving several variables (structural outliers), there is no difference in how the weighting scheme treats the outlying point. That is, the entire observation is either downweighted or not. Or still, all components of an observation are treated in the same way whether they are "responsible" for the outlyingness or not. This kind of weighting is of course intrinsic to all affine equivariant robust estimators. Indeed, once we start to treat the components of an observation differently, we have to give up the equivariance. However, by making this sacrifice we can make a distinction between contaminated and non-contaminated components of an observation. It thus allows us to downweight the contaminated components only, such that we avoid losing the information contained in the other components. We can then obtain an estimator with increased precision.

The Stahel-Donoho estimator, like other high-breakdown estimators, has been studied primarily in the context of the Tukey-Huber contamination model (Tukey (1962) and Huber (1964)). This model assumes that, on average, a large fraction $(1-\varepsilon)$ of the data (e.g. $0 \leq \varepsilon<0.25$ ) is generated from a classical model, whereas the remaining data can be affected by abnormal noise. In other words, the data come from a mixture distribution with a fully described dominant component (e.g. a Gaussian random variable) and an unspecified minority component. The general idea of robust procedures in this context is to conduct inference on the dominant component of the mixture, by limiting the influence of observations that resulted from the other component. Identifying and subsequently downweighting such "harmful" observations makes perfect sense for this purpose.

The Tukey-Huber model has some limitations, especially in high dimensions. The main criticisms are that it assumes that a majority of the points is per- 
fectly free of contamination, and that downweighting entire points may be inefficient. In Alqallaf et al. (2008), the Tukey-Huber model was extended to a large family of contamination models. One of these models, the independent contamination model, assumes that contamination in each variable is independent from the other variables, leading in particular to componentwise outliers. Furthermore, the models now merely assume that there is a majority of outlier-free values in each variable, but not necessarily a majority of outlier-free observations. Affine equivariant robust estimators do not perform well under such models because they require a majority of clean observations. The Stahel-Donoho estimator may seem somewhat better protected against a possible majority of outlying points than estimators such as MCD or Sestimators, as it is in principle allowed to downweight more than half of the points. Nevertheless the estimator surely is not particularly suitable for these models since it can not treat the variables separately. Downweighting a large number of points may lead to a considerable waste of information, especially in high dimensions, and may cause severe instability.

In this paper we investigate an adaptation of the Stahel-Donoho estimator where we allow separate weights for each component of an observation. The idea is to start from the outlyingness of the observation as measured in the original Stahel-Donoho procedure. Subsequently, for each observation, we attempt to identify to what extent each variable is contributing to the outlyingness and we use this information to adjust the original Stahel-Donoho weights in a cellwise manner. In particular, whenever an observation has a considerable outlyingness and hence a small Stahel-Donoho weight, the "clean" components should be restored to some extent by adjusting the corresponding weight upwards. By adapting the estimator in this way (using cellwise weights), we give up affine equivariance but we can gain precision. Moreover, the estimator becomes more suitable for use in the context of the larger family of contamination models considered in Alqallaf et al. (2008). That is, in case of independent contamination, the adaptation not only boosts the precision but may also increase the robustness.

The rest of the paper is organized as follows. In Section 2 we discuss the Stahel-Donoho estimator and focus on a real data set. In Section 3 we present our proposal for adapting the estimator in a componentwise manner. A simulation study is performed in Section 4, in which we compare several variants of our proposal and see to what extent they succeed in restoring the weights of the clean components of an observation while leaving the weights of the contaminated components unaffected. Section 5 investigates through a second simulation study specifically how the cellwise weights affect the precision and robustness of the estimator. We continue with real data examples in Section 6. Finally, Section 7 concludes and gives directions for further research. 


\section{Stahel-Donoho estimator}

Suppose $X=\left\{x_{1}, \ldots, x_{n}\right\} \subset \mathbb{R}^{p}$ is a set of $n$ observations. Let $\mu$ be a shift and scale equivariant univariate location statistic and let $\sigma$ be a shift invariant and scale equivariant univariate scale statistic. Then, for any $y \in \mathbb{R}^{p}$, the StahelDonoho outlyingness is defined as

$$
r(y, X)=\sup _{a \in S_{p}} \frac{\left|a^{\prime} y-\mu\left(a^{\prime} X\right)\right|}{\sigma\left(a^{\prime} X\right)}
$$

with $S_{p}=\left\{a \in \mathbb{R}^{p}:\|a\|=1\right\}$. This outlyingness measure is based on the idea that for any multivariate outlier, one can always find a one-dimensional projection for which the observation is a univariate outlier.

The Stahel-Donoho estimator of location and scatter $\left(T_{S D}, S_{S D}\right)$ is defined as

$$
T_{S D}=\frac{\sum_{i=1}^{n} w_{i} x_{i}}{\sum_{i=1}^{n} w_{i}}
$$

and

$$
S_{S D}=\frac{\sum_{i=1}^{n} w_{i}\left(x_{i}-T_{S D}\right)\left(x_{i}-T_{S D}\right)^{\prime}}{\sum_{i=1}^{n} w_{i}}
$$

where $w_{i}=w\left(r\left(x_{i}, X\right)\right)$ and $w: \mathbb{R}^{+} \rightarrow \mathbb{R}^{+}$is a weight function so that observations with large outlyingness get small weights (see Stahel (1981) and Donoho (1982)).

For $w$, we use the Huber-type weight function as advocated by Maronna and Yohai (1995). It is defined as

$$
w(r)=I_{(r \leq c)}+(c / r)^{2} I_{(r>c)},
$$

where $c$ is a threshold which we choose here as $c=\min \left(\sqrt{\chi_{p}^{2}(0.50)}, 4\right)$.

In order to attain maximum breakdown (see e.g. Maronna and Yohai (1995), Gather and Hilker (1997)) the univariate location statistic $\mu$ is set equal to the median (MED) and the scale statistic $\sigma$ is chosen to be the modified MAD, defined as

$\operatorname{MAD}^{*}\left(a^{\prime} X\right)=\frac{\left|a^{\prime} X-\operatorname{MED}\left(a^{\prime} X\right)\right|_{\left\lceil\frac{n+p-1}{2}\right\rceil: n}+\left|a^{\prime} X-\operatorname{MED}\left(a^{\prime} X\right)\right|_{\left(\left\lfloor\frac{n+p-1}{2}\right\rfloor+1\right): n}}{2 \beta}$

where $\beta=\Phi^{-1}\left(\frac{1}{2}\left(\frac{n+p-1}{2 n}+1\right)\right)$ and $x_{i: n}$ denotes the $i$ th order statistic of the data set. 
As an example, we consider the Philips data (Rousseeuw and Van Driessen (1999)). For each of 677 diaphragm parts for TV sets, nine characteristics were measured at the beginning of a new production line. In Figure 1 the StahelDonoho outlyingnesses of the Philips data are shown. It can be seen that observations 491 to 565 get a very large outlyingness and hence seem to be strongly deviating from the majority of the observations. Consequently, these

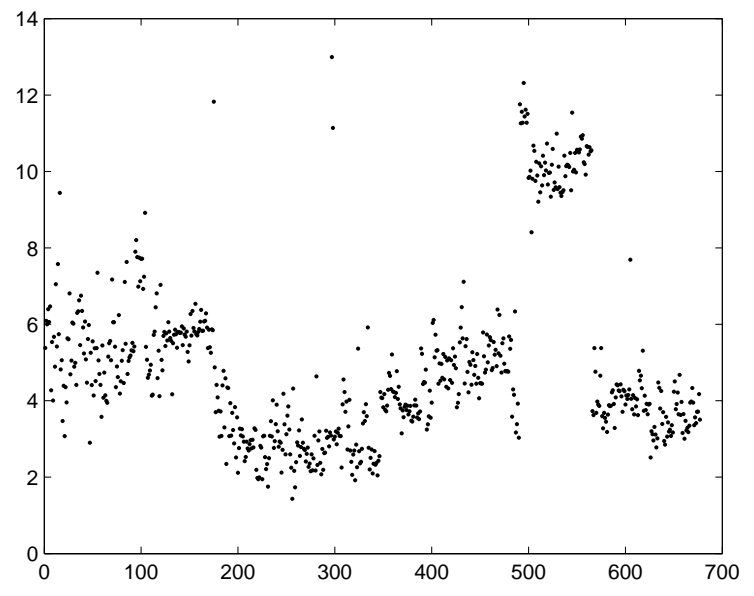

Fig. 1. Stahel-Donoho outlyingnesses of the Philips data.

aberrant observations will get a small weight in the computation of the StahelDonoho estimator, so that they influence the location and scatter estimate in a limited way. By giving a small weight to an outlying observation, every component of this observation will be downweighted. However, very often only a selected number of components is causing an observation to be outlying. The remaining components then have values that are in line with the majority of the data and do not contribute much to the large outlyingness. Therefore, perfectly valid information is potentially thrown away by downweighting the entire observation. Instead, one could attempt to incorporate componentwise information in the computation of the estimator.

In the Philips data, observations 491 to 565, among other points, are assigned a small weight in the Stahel-Donoho estimator. To assess whether it is truly required to downweight each component of these observations, we could first look at their outlyingness within each individual component. Figure 2 shows univariate scatter plots for each of the 9 components (V1, .., V9), standardized by their median and modified MAD. Some random jitter is added on the horizontal axis. Observations 491 to 565 are marked in dark. It can be seen, for example, that these observations are somewhat outlying within component V2. On the other hand, their values do not seem particularly suspicious within many of the other components, such as V1, V3, V4 or also V6.

Obviously, unsuspected behavior within individual components does not mean 
that the information contained in these components is harmless and can safely be used in the estimation of location and scatter of the data. Indeed, the large outlyingness of observations 491 to 565 could be due to the combined values of several components, rather than to those of individual components. To find out whether we are indeed dealing with such structural (or correlation) outliers, let us examine pairwise scatter plots. Figure 3(a) shows all pairwise plots involving component V1, while Figure 3(b) shows those involving component V6. Observations 491 to 565 are again marked in dark.

Regarding component V1, Figure 3(a) indicates that whenever some reasonable correlation structure is present between V1 and another component (which is the case for components V3 and V4, for example), observations 491 to 565 are well within the bulk of the data. It appears that component V1 contributes little to the outlyingness of these observations, even in combination with other components.

On the other hand for component V6, we see in Figure 3(b) that observations 491 to 565 are clearly outlying with respect to the correlation between V6 and V5, as well as that between V6 and V9. We may conclude that component V6 bears a considerable responsibility for the large outlyingness of observations 491 to 565 and hence should be downweighted when estimating location and scatter (even if these observations were not particularly outlying within the individual component V6).

Returning to component V1, based on Figure 3(a) we could decide that it should be safe to include this component of the outliers when computing the estimate. When the latter is supposed to be estimating the location and scatter of the "good" part of the data, its inclusion will probably not be harmful and is even likely to increase the precision of the estimate. In principle, however, before reaching such a conclusion we should go further and also examine threeway combinations of components, and so on. That would lead us too far in this example, but it will be taken into account in our proposals in the next

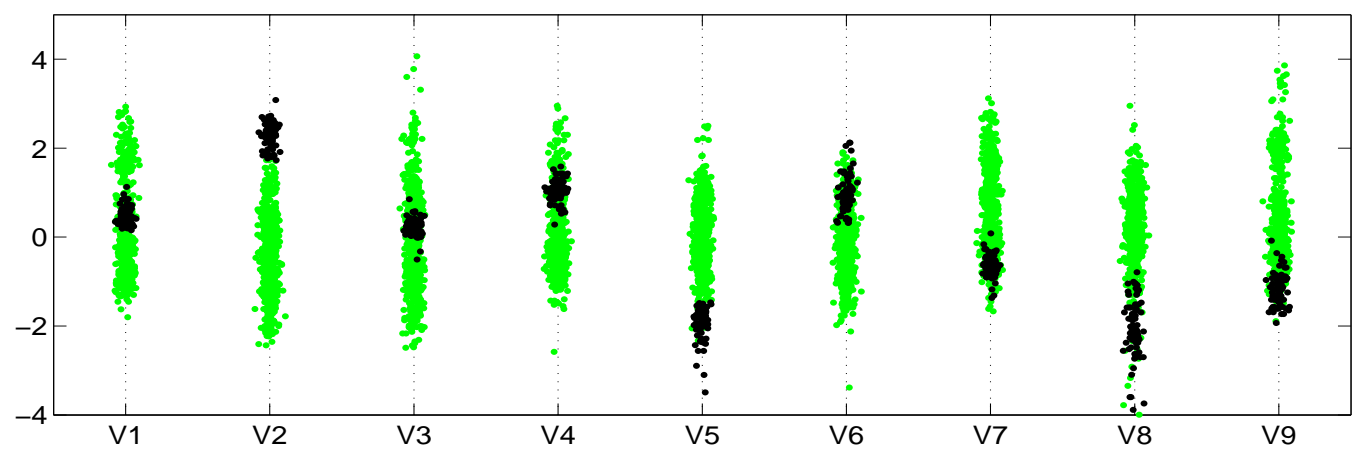

Fig. 2. Philips data, componentwise scatter: each component is standardized by its median and modified MAD. Observations 491 to 565 are marked in dark. 

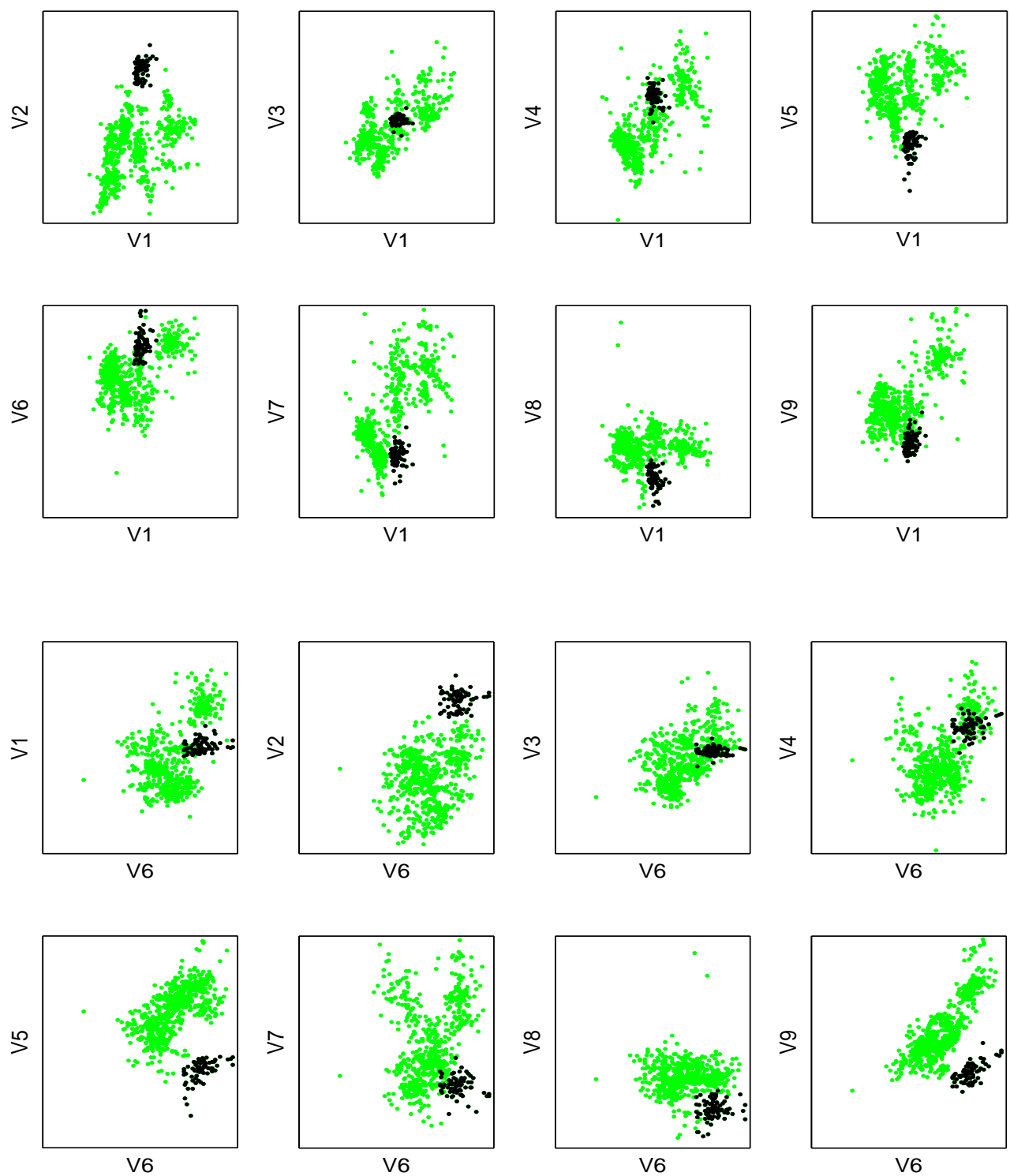

Fig. 3. Philips data, pairwise scatter plots: (a) all respective variables versus variable 1; (b) all respective variables versus variable 6. Observations 491 to 565 are marked in dark.

section.

\section{Adapted Stahel-Donoho estimators}

As mentioned before, a considerable amount of information could be wasted if we only have one scalar weight to control the influence of an observation on the 
Stahel-Donoho estimator. Therefore we propose an adaptation that assigns a vector of weights to each observation, i.e. every component of an observation receives its own weight.

Suppose $X=\left\{x_{1}, \ldots, x_{n}\right\} \subset \mathbb{R}^{p}$ is a set of $n$ observations. The adapted Stahel-Donoho estimator of location and scatter $\left(T_{S D^{*}}, S_{S D^{*}}\right)$ is defined as

$$
T_{S D^{*}, j}=\frac{\sum_{i=1}^{n} w_{i j} x_{i j}}{\sum_{i=1}^{n} w_{i j}}
$$

and

$$
S_{S D^{*}, j k}=\frac{\sum_{i=1}^{n} \sqrt{w_{i j}} \sqrt{w_{i k}}\left(x_{i j}-T_{S D^{*}, j}\right)\left(x_{i k}-T_{S D^{*}, k}\right)}{\sum_{i=1}^{n} \sqrt{w_{i j}} \sqrt{w_{i k}}}
$$

for $j, k=1, \ldots, p$.

The weight matrix $W=\left(w_{i j}\right)_{i j}$ is defined as

$$
w_{i j}=w\left(r_{i j}\right)
$$

where the weight function $w$ is as before and the adapted outlyingness $r_{i j}$ is defined as

$$
r_{i j}=\alpha_{i j} r_{i}+\left(1-\alpha_{i j}\right) c_{i j}
$$

where $\alpha_{i j}$ is a weighing parameter, $r_{i}$ is the Stahel-Donoho outlyingness of $x_{i}$ (i.e. $\left.r_{i}=r\left(x_{i}, X\right)\right)$ and $c_{i j}$ is the outlyingness of $x_{i}$ in the direction of component $j$, given by

$$
c_{i j}=\frac{\left|x_{i j}-\operatorname{MED}\left(X_{j}\right)\right|}{\operatorname{MAD}^{*}\left(X_{j}\right)} .
$$

Here, $X_{j}$ represents the set of $j$ th components of all observations in $X$ (i.e. $\left.X_{j}=\left\{x_{1 j}, \ldots, x_{n j}\right\}\right)$, MED is the median and MAD* is defined by (3).

The weighing parameter $\alpha_{i j}$ in (7) will be chosen in [0,1], such that the adapted outlyingness $r_{i j}$ is a weighted average of $r_{i}$ and $c_{i j}$. Since the componentwise directions are a subset of the directions considered in (1), we always have that $r_{i} \geq c_{i j}$ and hence $r_{i} \geq r_{i j}$. That is, the adapted outlyingness is smaller than the original outlyingness. The idea is to reduce the outlyingness, and hence increase the weight, of those components which had limited influence on the global outlyingness $r_{i}$ (i.e. those components for which a weight increase is "justified"), while largely keeping the original outlyingness and weight for the other components.

We could choose $\alpha_{i j}$ as a constant, i.e. $\alpha_{i j}=\alpha$, in which case the reduction in outlyingness associated with $r_{i j}$ is linearly related to $c_{i j}$, the outlyingness in the $j$ th component. This would make sense if we only suspect componentwise outliers but seems not appropriate for structural outliers. Indeed, if $x_{i}$ is such a structural outlier, it may have components with small $c_{i j}$ which nevertheless 
share a responsibility for the large $r_{i}$ and hence should not be awarded any considerable reduction in outlyingness. In order now to account for structural outliers as well, $\alpha_{i j}$ would preferably represent the extent to which the $j$ th component contributes to $r_{i}$. If this contribution is assessed to be large, $r_{i j}$ should remain close to $r_{i}$, otherwise $r_{i j}$ is allowed to decrease largely towards $c_{i j}$.

In this paper we consider the following choices for $\alpha_{i j}$ :

(1) $\alpha_{i j}=\alpha=1 / 2$. Hence the adapted outlyingness $r_{i j}$ always equals the average of $r_{i}$ and $c_{i j}$. As explained above, a constant weighing parameter may not account appropriately for structural outliers. Another disadvantage is that the choice of $\alpha=1 / 2$ is arbitrary and may be far from optimal in some situations. We do not expect this choice of weighing parameter to perform well, but we consider it as a benchmark.

(2) $\alpha_{i j}=\left(\max _{k=1}^{p} c_{i k}\right)^{-1} c_{i j}$ with $c_{i j}$ as defined in (8). It follows that $\alpha_{i j}$ is large whenever $c_{i j}$ is large, relative to the outlyingnesses in the direction of the other components. This weighing parameter $\alpha_{i j}$ compares to a constant $\alpha$ as follows: (1) in case observation $x_{i}$ is a componentwise outlier, the contrast between the $r_{i j}$ of contaminated and non-contaminated components is now stronger; (2) in case $x_{i}$ is a structural outlier involving multiple components, all of the $c_{i j}$ may be relatively small and then this choice of $\alpha_{i j}$ is more conservative and may avoid unwarranted reduction of outlyingness for those components sharing responsibility for the large outlyingness $r_{i}$.

(3) $\alpha_{i j}=\left(\max _{k=1}^{p}\left|u_{i k}\right|\right)^{-1}\left|u_{i j}\right|$ where $u_{i}=\left(u_{i 1}, \ldots, u_{i p}\right)$ denotes the direction that maximizes $r_{i}$. Hence, $\alpha_{i j}$ is large whenever the $j$ th component has a relatively large coefficient in the maximizing direction $u_{i}$. The underlying idea is that the magnitude of the coefficients in $u_{i}$ reflects the extent to which the respective components are responsible for the outlyingness $r_{i}$, both in case of componentwise and structural outliers.

We will refer to these methods as (1) SDH, (2) SDC and (3) SDM (respectively from Half, Components and Maximizing). The resulting location and scatter estimates (4) and (5) will sometimes be denoted by $\left(T_{S D H}, S_{S D H}\right)$, $\left(T_{S D C}, S_{S D C}\right)$ and $\left(T_{S D M}, S_{S D M}\right)$ respectively. Note further that when $\alpha_{i j}=1$, we obtain the original Stahel-Donoho estimator $\left(T_{S D}, S_{S D}\right)$.

Remark: when applying the SDM method, it would be desirable to rescale each variable first by dividing it componentwise by its MAD, ensuring that the variances of the components are within the same range. This would avoid that the maximizing directions $u_{i}$ are attracted to the variables with the smallest scales. Note that rescaling the variables by the componentwise MAD before 
computing the maximizing directions $u_{i}$, however, is equivalent to adjusting the directions $u_{i}$ by multiplying its coefficients by the respective MADs (without rescaling the variables). In the rest of the paper, adjusted $u_{i}$ vectors will be used in the SDM method.

\section{Simulation study}

In this section we investigate through simulation to what extent our methods succeed in reducing the outlyingness, and thus increasing the weight, of precisely those components for which it would be justified.

Samples $\left\{x_{1}, \ldots, x_{n}\right\}$ of size $n=100$ in $p=3$ dimensions were generated from a standard normal distribution $N(0, \Sigma)$. For each sample, $100 \epsilon \%$ of the data were shifted over a distance of $k \mathbf{m}$. (Additionally, the variance was reduced for those components with non-zero coefficient in the outlying direction $\mathbf{m}$, by multiplying their standard deviation by 0.1 ).

We considered two choices for $\Sigma$, respectively corresponding to uncorrelated and partly correlated data: (1) $\Sigma_{u}=I_{3}$ (i.e. the identity matrix), and (2)

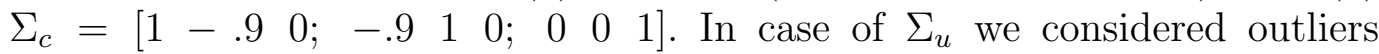
in directions $\mathbf{m}=(1,0,0),(1,1,0)$ and $(1,1, u)$ with $u \geq 1$. For the correlated data corresponding to $\Sigma_{c}$ we looked at outliers in directions $\mathbf{m}=$ $(1,0,0),(0,0,1),(1,1,0),(1,0,1),(1,1, u)$ and $(1, u, 1)$, again with $u \geq 1$. The distance $k$ and the proportion $\epsilon$ of the outliers were varied.

For each situation, 100 samples were generated. For each sample, and for each observation $x_{i}$, we computed the Stahel-Donoho outlyingness $r_{i}$ and subsequently the adapted outlyingnesses $r_{i j}$ based on the methods SDH, SDC and SDM. The corresponding weights $w_{i}$ and $w_{i j}$ are based on the weight function (2). When the Stahel-Donoho procedure is sufficiently robust against the outliers generated as above, the weight $w_{i}$ will be close to 0 for the outliers and close to 1 for the regular observations. The adaptations SDH, SDC or SDM then can be said to work appropriately if the following more or less holds:

(1) if $x_{i}$ was generated as an outlier, and if the $j$ th component has a zero coefficient in the outlying direction $\mathbf{m}$, then $w_{i j}$ is high (i.e. the weight of the $j$ th component should be awarded an increase compared to the SD weight).

(2) if $x_{i}$ was generated as an outlier, and if the $j$ th component has a nonzero coefficient in the outlying direction $\mathbf{m}$, then $w_{i j}$ is close to $w_{i}$ (i.e. the weight of the $j$ th component should be similar to the original SD weight).

(3) if $x_{i}$ was generated as a regular observation, then $w_{i j}$ is high (i.e. the 
weights of all components should be as close as possible to 1).

(Recall that by definition $w_{i j} \geq w_{i}$.) When reporting our results we will divide the components $x_{i j}$ into groups corresponding to these three situations, which we will also respectively refer to as (1) non-contaminated components of outliers; (2) contaminated components of outliers; and (3) components of regular observations. In particular we will report the mean weights $w_{i}$ and $w_{i j}$ for each of the three groups, over all generated samples in each of the outlier situations described above.

For the data in this simulation study, we would say that we are dealing with componentwise outliers as soon as the distance $k$ is sufficiently large (such that $c_{i j}$ is large for each contaminated component $j$ ). On the other hand, we would refer to structural outliers for those cases where each $c_{i j}$ is relatively small but the global outlyingness $r_{i}$ is large. This especially occurs in case of covariance matrix $\Sigma_{c}$ and outliers in the direction $(1,1,0)$ or $(1,1, \mathrm{u})$ at a relatively small distance $k$. However, we do not attempt to make a strict distinction between the two types of outliers in this study. In fact we make the general assumption that those components that were not contaminated, i.e. that were actually generated according to the standard normal distribution, are justified to have their weight increased. The other components are not.

Note that exact computation of the supremum in the Stahel-Donoho outlyingness (1) is impractical and typically a random search algorithm based on subsampling is used to obtain an approximation. In this paper we applied a Matlab implementation of the Gauss-algorithm used in Maronna and Yohai (1995). The number of random directions in the algorithm was taken equal to 1000, which should be sufficient for $p$ as small as in this simulation study (see Maronna and Yohai 1995).

We first consider the simple case of $\Sigma=\Sigma_{u}$, such that none of the components are correlated. In this situation all outliers may be regarded as componentwise outliers. The top panel in Figure 4 presents the results for $\mathbf{m}=(1,0,0)$, while the bottom panel represents the case $\mathbf{m}=(1,1, u)$. The results for $\mathbf{m}=(1,1,0)$ are omitted as they are very similar to the case $\mathbf{m}=(1,0,0)$. Here, and in the following, the left plots correspond to the group of noncontaminated components of outliers, the middle plots to the contaminated components, and the right plots to the components of regular observations. Each plot shows the mean values of $w_{i}$ and $w_{i j}$ of all concerned components in all generated samples. The top row of a panel always presents the results as a function of the outlier location (determined by $k$ and/or $u$ ) for fixed proportion of outliers $\epsilon$. In the bottom row the outlier location ( $k$ and/or $u$ ) is fixed and the plots show the weights in function of the fraction of outliers $\epsilon$. The solid lines correspond to the initial SD weights $w_{i}$, while the dashed, dotted and dash-dotted lines respectively represent the SDH, SDM and SDC 


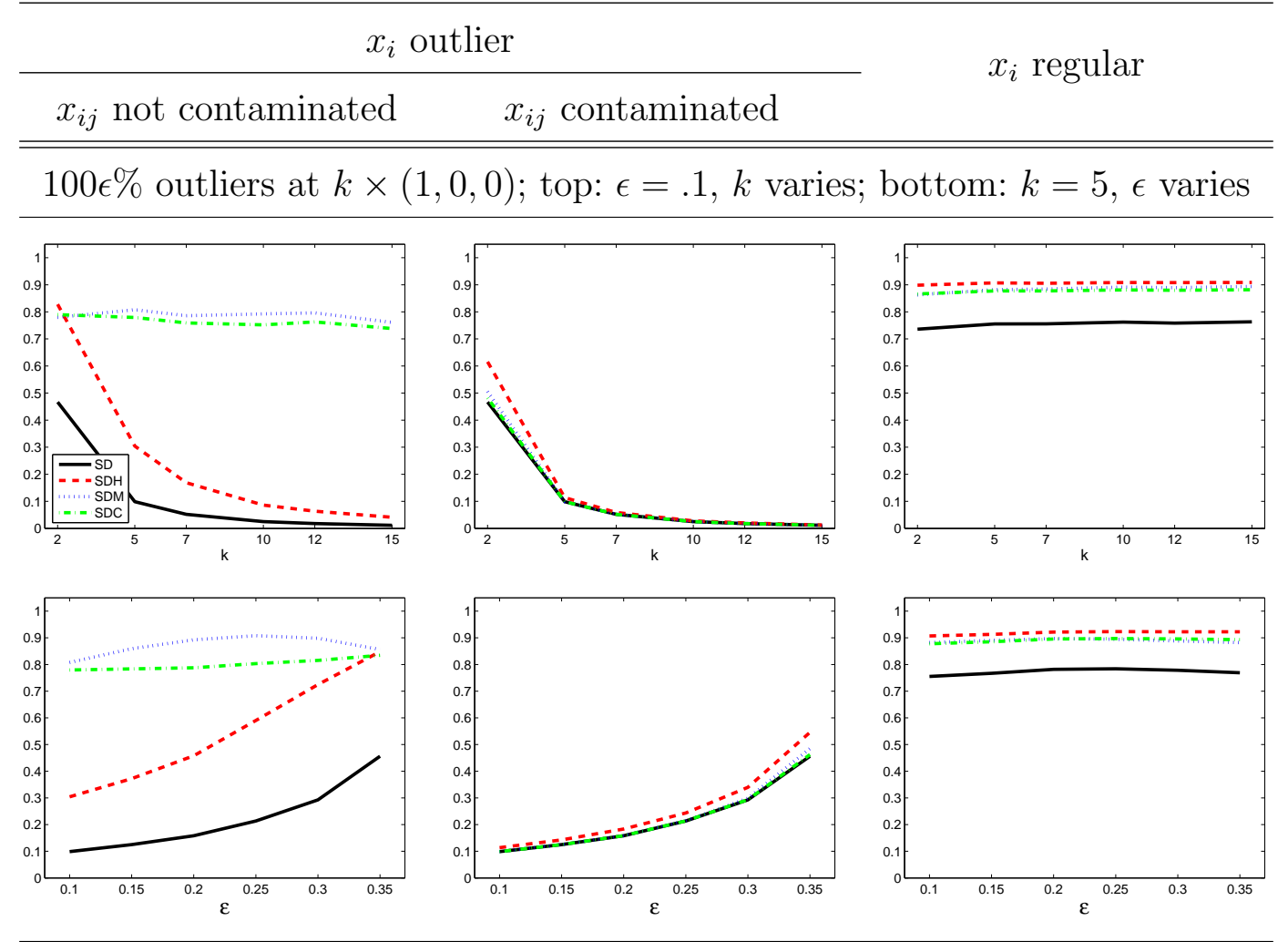

$100 \epsilon \%$ outliers at $5 \times(1,1, u)$; top: $\epsilon=.1, u$ varies; bottom: $u=5, \epsilon$ varies

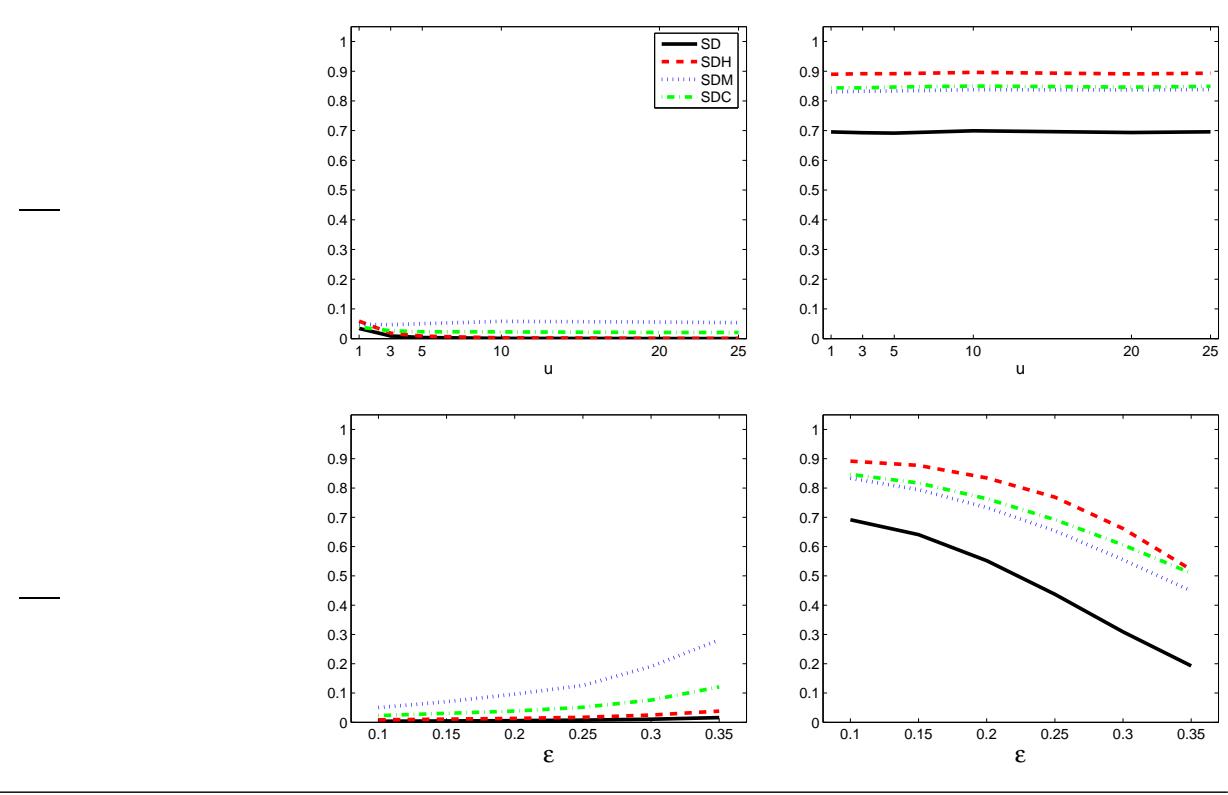

Fig. 4. Simulation results uncorrelated data: mean weights for (left) non-contaminated components of outliers, (middle) contaminated components of outliers, (right) components of regular observations; various outlier configurations as indicated

weights $w_{i j}$.

Consider the choice $\mathbf{m}=(1,0,0)$, i.e. outliers are situated in the first compo- 
nent only, in the top panel of Figure 4. In the top row, where $\epsilon=.1$ and the distance $k$ varies, we see that the SD weights for the outliers are close to 0 , except when $k$ is small in which case the Stahel-Donoho estimator hence could not withstand the outliers. For larger $k$ we see in the left plot that the SDC and SDM method are both succesful in awarding an increase in weight for the non-contaminated components of the outliers. SDH succeeds in only a limited increase. From the middle plot it is clear that all methods leave the weight of the contaminated component almost untouched, which is as desired. The right plot, representing the regular observations, indicates that all methods yield a slight gain in precision by somewhat increasing the (already high) average weights of those observations. Note that increasing the weight of clean components may constitute a gain in robustness, rather than a gain in precision, if it offsets a possible bias induced by contamination in the corresponding components from other observations (see the next section).

In the bottom row of the top panel in Figure 4 the distance of the outliers is fixed at $k=5$ and the proportion $\epsilon$ is varied from $10 \%$ to $35 \%$. It is again clear that the non-contaminated components obtain a considerable weight increase in case $w_{i}$ is low, while the contaminated components do not. We may conclude that in case of $\mathbf{m}=(1,0,0)$, the adapted outlyingnesses succeed nicely in boosting precision while largely preserving robustness.

Now for the choice $\mathbf{m}=(1,1, u)$, which is shown in the bottom panel of Figure 4 , each component of the outliers is contaminated and hence we have only two plots in each row of this panel. Our interest is now primarily in the components of the outliers, which preferably should not be awarded an increase in weight. The main difficulty for our methods here (especially SDC and SDM) is that the contamination in components 1 and 2 would be dwarfed by the much more severe contamination in component 3 , and that this may lead to the first two components obtaining a somewhat unjustified gain in weight. However, because it always holds that $r_{i} \geq c_{i j}$ it follows from (7) that also the adapted outlyingness $r_{i j}$ is bounded below by $c_{i j}$. Hence, whenever an observation is outlying in the direction of the $j$ th component, any decrease in outlyingness awarded to that component is bounded by the large componentwise outlyingness $c_{i j}$. Therefore, any gain in weight $w_{i j}$ is kept limited. This ensures that our methods to a reasonable extent are conservative, which indeed can be seen in the results in Figure 4.

The top row again takes $\epsilon=.1$, fixes additionally $k=5$, and shows the mean weights for a grid of increasing $u$. The bottom row sets $k=5$ and $u=5$ and varies the proportion of outliers. First, the SDH method has a fixed weighing parameter $\alpha_{i j}$ and that is the reason why it fares reasonably well in this situation. Indeed, its weights $w_{i j}$ are closest to the SD weights $w_{i}$ on average. Both SDM and SDC yield a somewhat larger increase for the weights of the contaminated components, which can be entirely attributed to 
the components 1 and 2 , for which $\alpha_{i j}$ is relatively low compared to component 3. Nevertheless, the increase in weight is very limited, for reasons explained above, and it is not deemed harmful in the sense that at worst it would yield a slight bias increase in the adapted Stahel-Donoho estimates. In the next section, we will examine the issue of robustness and possible bias increase of the presented estimators more thoroughly.

Next, we consider the partly correlated data generated with $\Sigma=\Sigma_{c}$. The situation is now more complicated since the high negative correlation between components 1 and 2 could produce far outliers for which none of the components needs to be severely contaminated. Or, in other words, the SD outlyingness $r_{i}$ is potentially much higher than any of the componentwise $c_{i j}$. Figure 5 contains the results for $\mathbf{m}=(1,0,0)$ and $(1,1,0)$, while Figure 7 contains those for $\mathbf{m}=(1,0,1)$ and $(1,1, u)$. Results for $\mathbf{m}=(0,0,1)$ and $(1, u, 1)$ are omitted (these results were found to be comparable to respectively $\mathbf{m}=(1,0,0)$ and $(1,1, u)$ in case of uncorrelated data, as considered above).

Let us first look at the results for $\mathbf{m}=(1,0,0)$ in the top panel of Figure 5 . The two rows again respectively fix the outlier proportion at $\epsilon=.1$ and the distance at $k=5$. We see that the behavior of the methods looks similar to that in the corresponding uncorrelated setting that was shown in Figure 4, top panel, except for the following differences. First, note that the SD weights are generally lower than in Figure 4 because the outliers now are more pronounced and hence easier to detect. Indeed, although the outliers were generated in the first component only, because of the high correlation the outlyingness $r_{i}$ becomes considerably higher (and is maximized) in a direction which additionally involves the second component. This is also the reason why, as seen in the left plots, the SDM method behaves somewhat more conservative for the non-contaminated components than in the uncorrelated setting. In fact, the dashed curves are rather low here because the second component is considered by the SDM $\alpha_{i j}$ parameter as partly responsible for the high $r_{i}$ and hence does not receive a high weight. The third component on the other hand does generally receive a gain in weight and so the SDM curves still end up somewhere in the middle of 0 and 1 . The fact that SDM wrongly attributes some responsibility to the second component is understandable and difficult to avoid, since the data correlation implies that these particular outliers could equally easily have been produced by contaminating both components 1 and 2, as shown in Figure 6. Regarding SDC, the method comes out on top but its performance for non-contaminated components is also somewhat worse than in Figure 4. The reason is that $r_{i}$ is now much higher, due to the correlation, while the weighing parameter $\alpha_{i j}$ remains the same. For the contaminated components shown in the middle plots, the methods perform very well, except for SDH which seems overly liberal here. Hence, we may again conclude that SDC and SDM best preserve the outlier resistance while increasing the precision. 


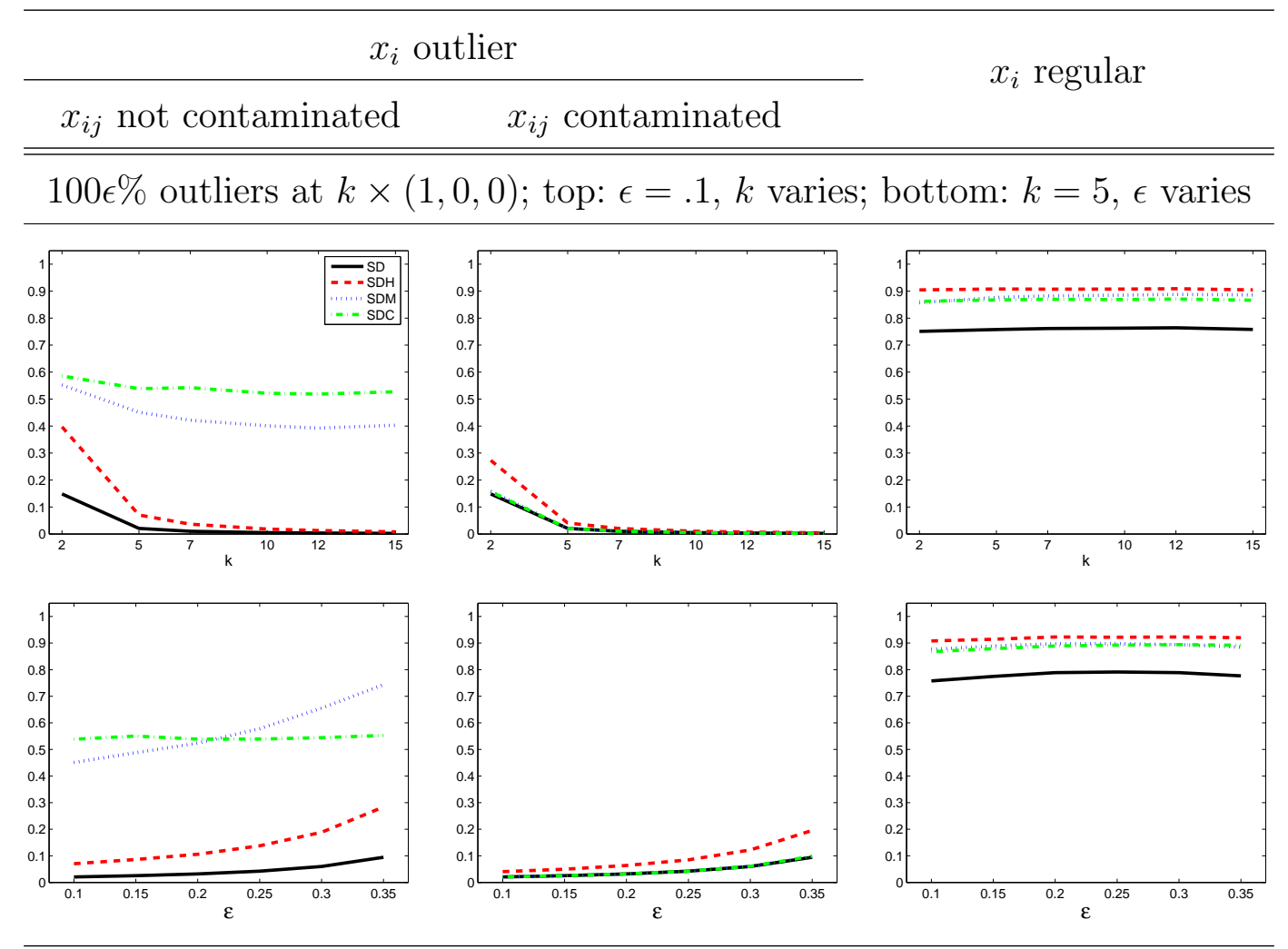

$100 \epsilon \%$ outliers at $k \times(1,1,0)$; top: $\epsilon=.1, k$ varies; bottom: $k=5, \epsilon$ varies
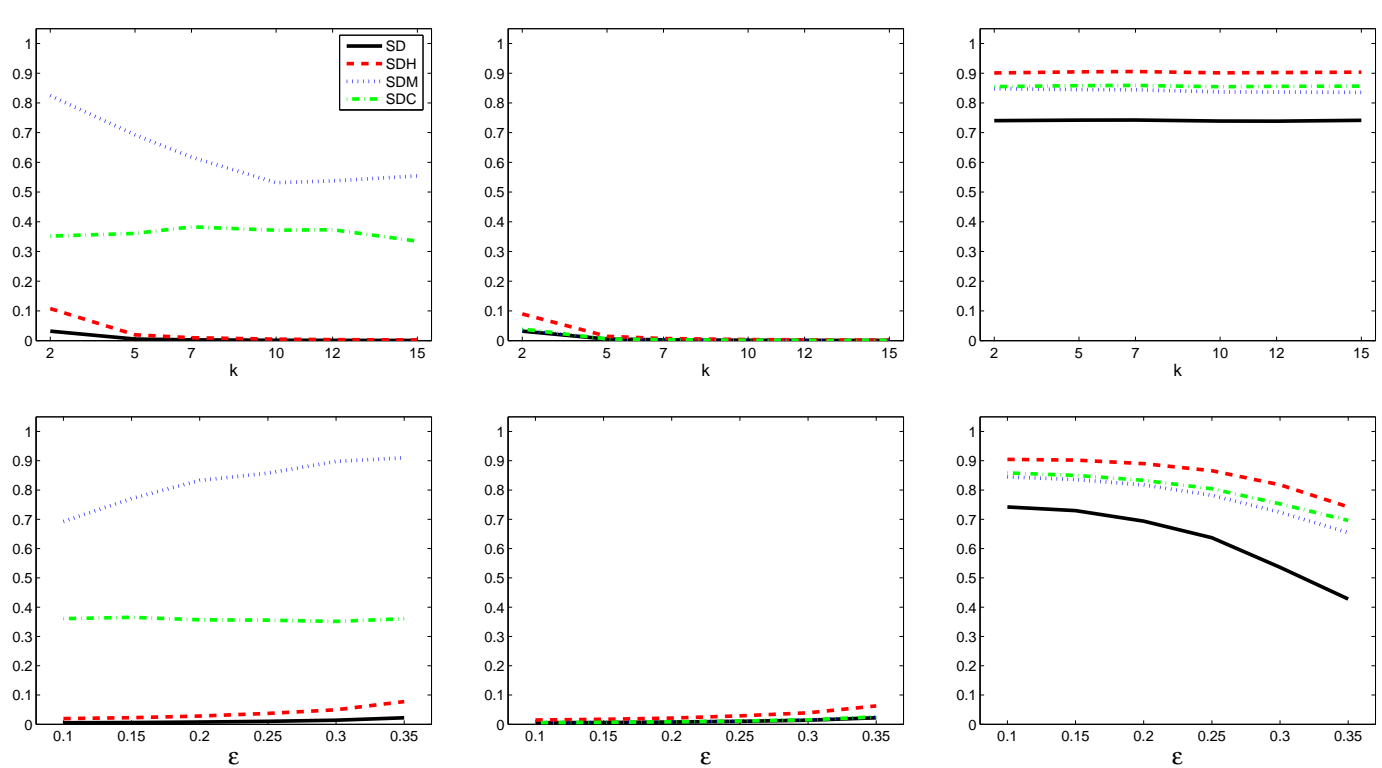

Fig. 5. Simulation results correlated data: mean weights for (left) non-contaminated components of outliers, (middle) contaminated components of outliers, (right) components of regular observations; various outlier configurations as indicated

The bottom panel in Figure 5 corresponds to outliers in the direction $\mathbf{m}=$ $(1,1,0)$, which means that the outliers are now even more pronounced than in the previous setting, given $k$, resulting in even lower SD weights $w_{i}$. The only 

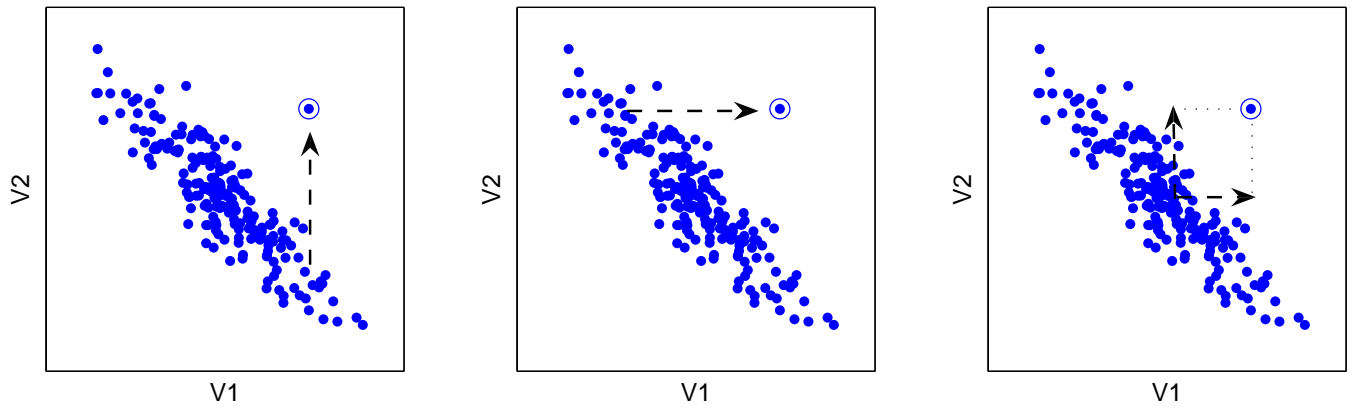

Fig. 6. 2-D example with highly correlated data: different causes leading to the same outlier

non-contaminated component of the outliers is (the uncorrelated) component 3 , and we see in the left plots that SDM performs best in awarding this component a higher weight. The SDC method clearly has difficulties to overcome the large $r_{i}$ in view of the small $c_{i j}$ (and the small differences among the $c_{i j}$ ) and therefore turns out to be more conservative here. The SDH method, finally, completely fails as it is unable to distinguish properly between contaminated and non-contaminated components (especially in case of small $k$ ).

Next, consider the case $\mathbf{m}=(1,0,1)$ in the top panel of Figure 7 , where the only non-contaminated component is the second one, which is of course highly negatively correlated with the first one. We see in the left plots that the SDM method hardly increases the weight of the second component, because it deems the latter partly responsible for the outlyingness of these observations. The current situation is comparable to the one corresponding to $\mathbf{m}=(1,0,0)$ in Figure 5, top panel, where we had additionally component 3 as non-contaminated (which ensured that the SDM curves on average still indicated an increase). The SDC method here again performs well, similarly to the $\mathbf{m}=(1,0,0)$ case.

Finally, the bottom panel of Figure 7 represents outliers in $\mathbf{m}=(1,1, u)$. The top row fixes $\epsilon=.1$ and $k=2$, and shows the mean weights in function of $u$. The bottom row varies again the proportion of outliers, with $k=2$ and $u=5$. Since all components of the outliers are contaminated we again have only two plots to consider. Note that the small $k$ in combination with the high correlation implies that we are dealing with truly structural outliers. We are concerned here that the components of the outliers should not receive a high weight $w_{i j}$. We see, however, that SDC is behaving in a way which is somewhat too liberal in this context. The reason is that it believes it should justifiably increase the weights of components 1 and 2 since their $c_{i j}$ is very small compared to that of component 3. The SDM method does better in this regard.

This last situation illustrates the main difficulty of our methods, namely that 


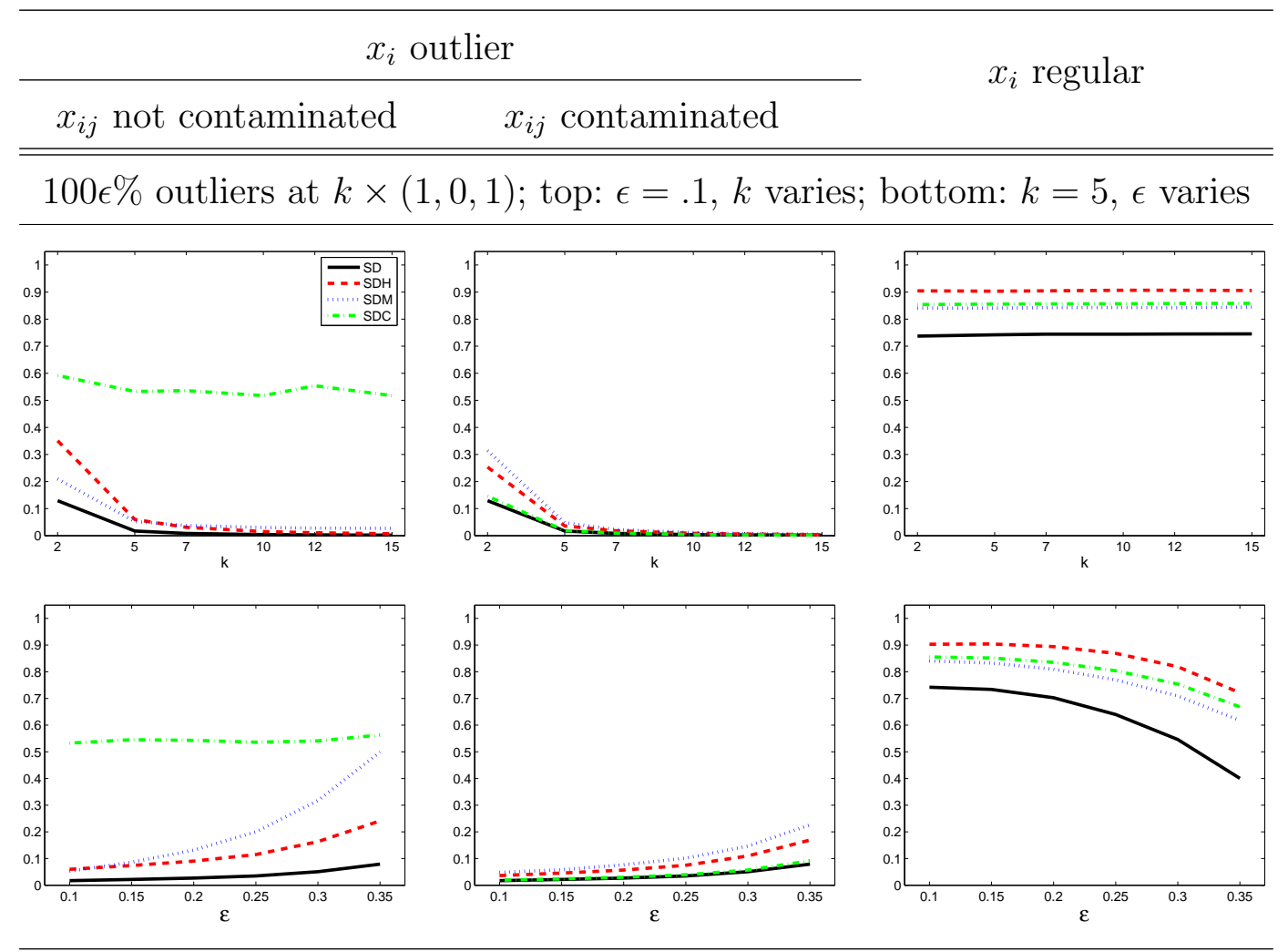

$100 \epsilon \%$ outliers at $2 \times(1,1, u)$; top: $\epsilon=.1, u$ varies; bottom: $u=5, \epsilon$ varies

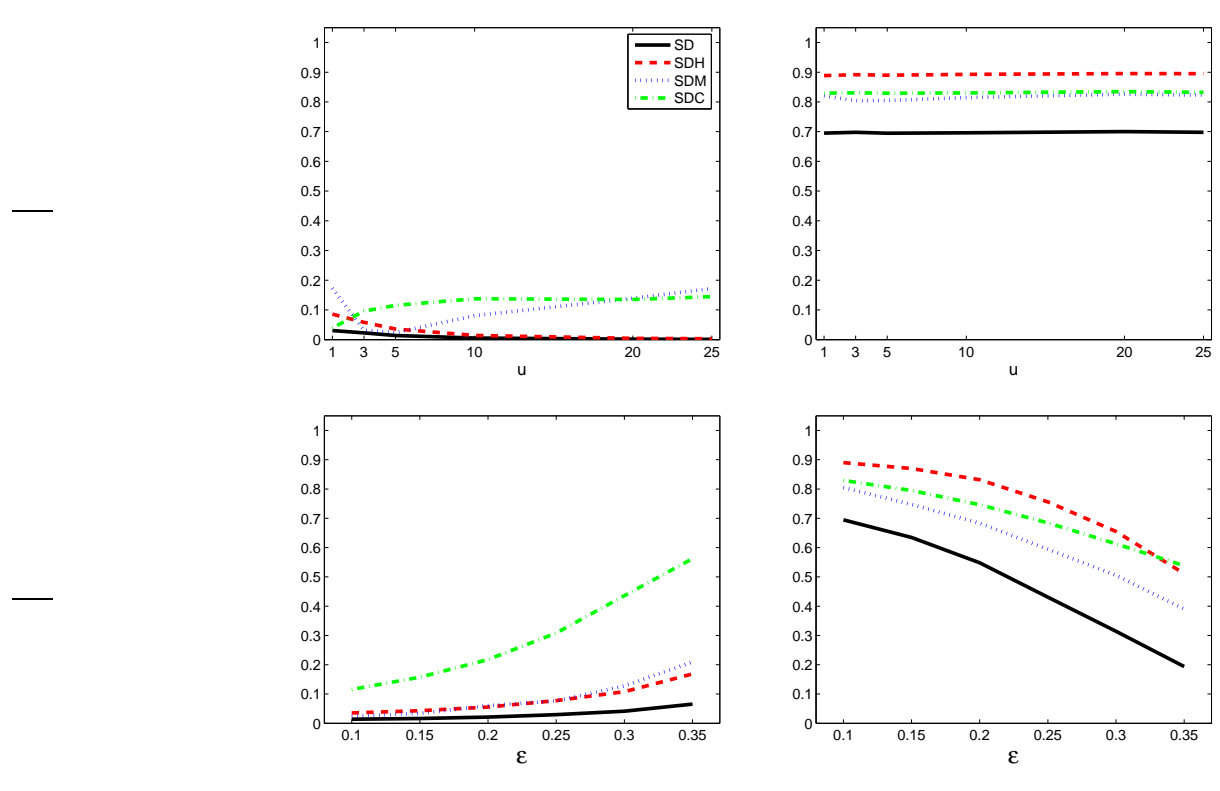

Fig. 7. Simulation results correlated data: mean weights for (left) non-contaminated components of outliers, (middle) contaminated components of outliers, (right) components of regular observations; various outlier configurations as indicated

they may be underestimating the responsibility of some components in case of certain structural outliers, e.g. in case of large outlyingness due to correlation combined with other outlying components. In general, we may con- 
clude that both SDC and SDM offer a considerable increase in weights for non-contaminated components for many outlier situations. In some cases this increase in weights also leads to an increased robustness, such as in the case of independent contamination in the different components. However, in other cases the potential gain in precision comes at a cost of increased weights for contaminated components, and thus some loss of robustness, but this cost is limited since the adjusted outlyingnesses $r_{i j}$ are bounded by the componentwise outlyingnesses $c_{i j}$. In the next section we examine more closely this possible trade-off between precision and robustness.

\section{$5 \quad$ Precision and robustness}

In this section we show the results of a simulation study that investigates how the precision and the robustness of the Stahel-Donoho estimator are affected by our adaptations. In particular we present mean squared errors (MSEs) of the estimates, and we focus on the location part. Here, precision is meant to represent the variance of the estimates, while by robustness we mainly refer to the bias.

Recall that our adaptations can only adjust the weights $w_{i j}$ of the observations upward from the Stahel-Donoho weight $w_{i}$. Such upward adjustments produce either a reduction or an increase of the MSE for the resulting location estimates $T_{S D^{*}, j}$, depending on which of the three groups (as distinguished in the previous section) component $x_{i j}$ belongs to:

(1) non-contaminated components of outliers: reduction of the MSE, due to gain in precision or bias reduction

(2) contaminated components of outliers: increase of the MSE, due to a bias increase

(3) components of regular observations: reduction of the MSE, due to gain in precision or bias reduction

Regarding the reduction of the MSE, we distinguish between gain in precision and bias reduction. The former refers to a variance reduction in case the original SD estimate $T_{S D, j}$ was roughly unbiased (either because there are no harmful outliers in the $j$-th component or the outliers have been downweighted by the SD estimate). Bias reduction, on the other hand, corresponds to the situation where $T_{S D, j}$ is biased because of outliers that have not been sufficiently downweighted. In such cases, adjusting the weight $w_{i j}$ for an observation in which the $j$-th component is clean, indeed may reduce the bias of $T_{S D, j}$.

Hence, the interest in this study is essentially to investigate whether the positive MSE effect corresponding to components from (1) and (3) outweighs the 
negative MSE effect of observations from (2), resulting in an average MSE reduction.

Samples $X=\left\{x_{1}, \ldots, x_{n}\right\}$ were generated from a $p$-variate normal distribution, with $p$ taking the values 5 and 10, and size $n=10 p$. Subsequently, contamination was added.

\subsection{Componentwise outliers}

First, we used a standard normal distribution and randomly added univariate outliers, independently in each component. In particular, for component $X_{j}$ (with $j=1, \ldots, p$ ), $100 \varepsilon \%$ of the observations $\left\{x_{1 j}, \ldots, x_{n j}\right\}$ were shifted over a distance of $k m_{j}$, with $m_{j}$ the $j$-th component (after normalization) of the outlying direction $\mathbf{m}$. Again, the variance was reduced for those components with non-zero coefficient in $\mathbf{m}$, by multiplying their standard deviation by 0.1 .

We considered various choices of $\mathbf{m}$, with outlying distances $k=6,24,64$ and 160. For each situation, $N=500$ samples were generated. Then, for each sample $X^{(l)} ; l=1, \ldots, N$ and for each observation $x_{i}$ in $X^{(l)}$, we computed the Stahel-Donoho outlyingness $r_{i}$, the adapted outlyingnesses $r_{i j}$ based on the methods SDH, SDC and SDM, and subsequently the corresponding location estimates $T_{., j}^{(l)}$. The number of random directions in the algorithm was set at $200 p$.

Next, the MSE of the various methods was calculated as

$$
\operatorname{MSE}(T .)=\underset{j=1, \ldots, p}{\operatorname{ave}}\left(\underset{l=1, \ldots, N}{\operatorname{ave}}\left(T_{., j}^{(l)}\right)^{2}\right)
$$

To simplify interpretation, the MSE was computed also separately for the variables which contain contaminated observations and the variables without any contamination:

$$
\operatorname{MSE}_{C}(T .)=\underset{j \in C}{\operatorname{ave}}\left(\underset{l=1, \ldots, N}{\operatorname{ave}}\left(T_{., j}^{(l)}\right)^{2}\right), \quad \operatorname{MSE}_{N C}(T .)=\underset{j \in N C}{\operatorname{ave}}\left(\underset{l=1, \ldots, N}{\operatorname{ave}}\left(T_{., j}^{(l)}\right)^{2}\right)
$$

with $C$ and $N C$ respectively denoting the set of variables with and without contamination.

We first consider the case $p=5$ where $20 \%$ of independent contamination was added in the outlying direction $\mathbf{m}=(1,1,0,0,0)$. The top row in Figure 8 presents the MSE for the location estimates of the original SD (solid) and the adaptations SDH, SDM and SDC (dashed, dotted and dash-dotted respectively), for different values of the outlying distance $k$. From these plots, it can be seen that the adjusted methods SDH, SDM and SDC perform well, 
$p=5, n=50,20 \%$ outliers at $k \times(1,1,0,0,0) ; k$ varies; top: total MSE;

bottom: MSE of contaminated $(\mathrm{C})$ and non-contaminated $(\mathrm{NC})$ variables
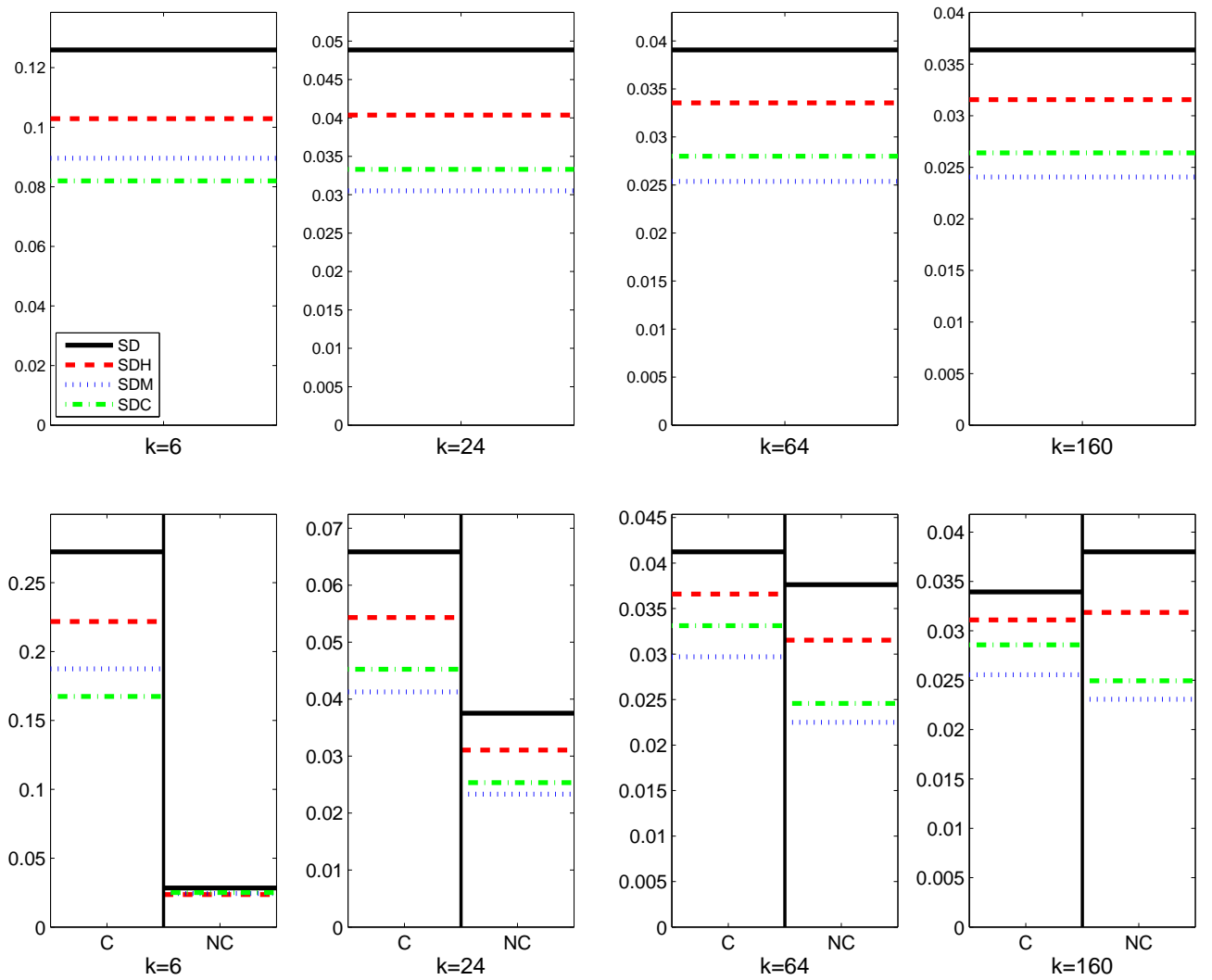

Fig. 8. Simulation results univariate outliers: mean squared errors for different $k$ values; various outlier configurations as indicated

as their overall MSE is considerably smaller than the MSE of the original Stahel-Donoho estimator.

To get more insight in this MSE reduction, we split up the MSE by looking at the contaminated and non-contaminated variables separately. As contamination was added in direction $(1,1,0,0,0)$ here, only variables 1 and 2 are contaminated. The average MSE for these two components of the location estimate, $\mathrm{MSE}_{C}$, is shown in the left column (C) of the plots in the bottom row of Figure 8. The MSE of the remaining components, $\mathrm{MSE}_{N C}$, is shown in the right column (NC).

First, it is clear that the original SD estimate suffers from a bias due to the contamination in the first two components, which corresponds to $\mathrm{MSE}_{C}$ being larger than $\mathrm{MSE}_{N C}$. The bias gradually disappears when the outlier distance $k$ increases, as the estimate succeeds better in downweighting the outliers. For small $k$ values, we see that the observed overall MSE reduction of our adaptations was mainly due to a reduction of $\mathrm{MSE}_{C}$. This particular reduc- 
$p=5, n=50,35 \%$ outliers at $k \times(1,1,0,0,0) ; k$ varies; top: total MSE;

bottom: MSE of contaminated $(\mathrm{C})$ and non-contaminated $(\mathrm{NC})$ variables
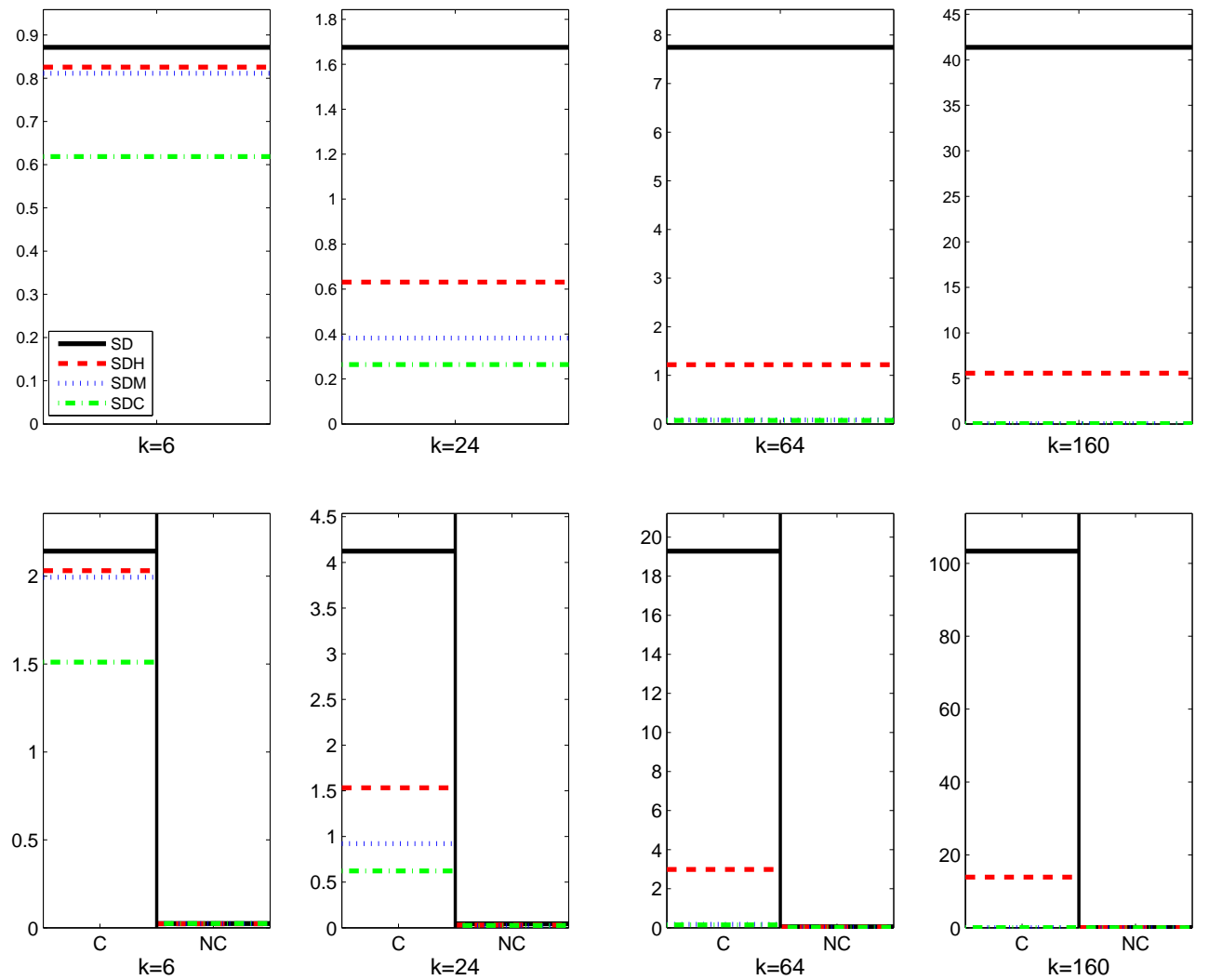

Fig. 9. Simulation results univariate outliers: mean squared errors for different $k$ values; various outlier configurations as indicated

tion results from observations in group (1) and (3) above receiving considerably higher weights and hence reducing the bias caused by the observations from group (2). The largest effect can be seen for SDC and SDM, which indicates that these methods succeed well in distinguishing contaminated from non-contaminated components. Regarding the non-contaminated components, where $\mathrm{MSE}_{N C}$ roughly corresponds to the variance, we see that SDC and SDM yield a relatively large gain in precision. For small values of $k$ however, this effect is somewhat dwarfed by the bias reduction in the contaminated components. For $20 \%$ of contamination in direction $(1,5,0,0,0)$ (results not shown) we obtained similar conclusions.

Figure 9 presents the results for $35 \%$ of independent contamination in the outlying direction $(1,1,0,0,0)$. We see that the original SD estimate is not able to resist this large amount of contamination, resulting in a very high MSE. However, the MSE of the adaptations SDH, SDM and SDC is rather low and hence offers a large improvement. Especially method SDC performs very well, with a huge bias reduction for the contaminated components. For 
$p=10, n=100,10 \%$ outliers at $k \times(1,1,1,1,1,0,0,0,0,0) ; k$ varies; top: MSE;

bottom: MSE of contaminated $(\mathrm{C})$ and non-contaminated $(\mathrm{NC})$ variables
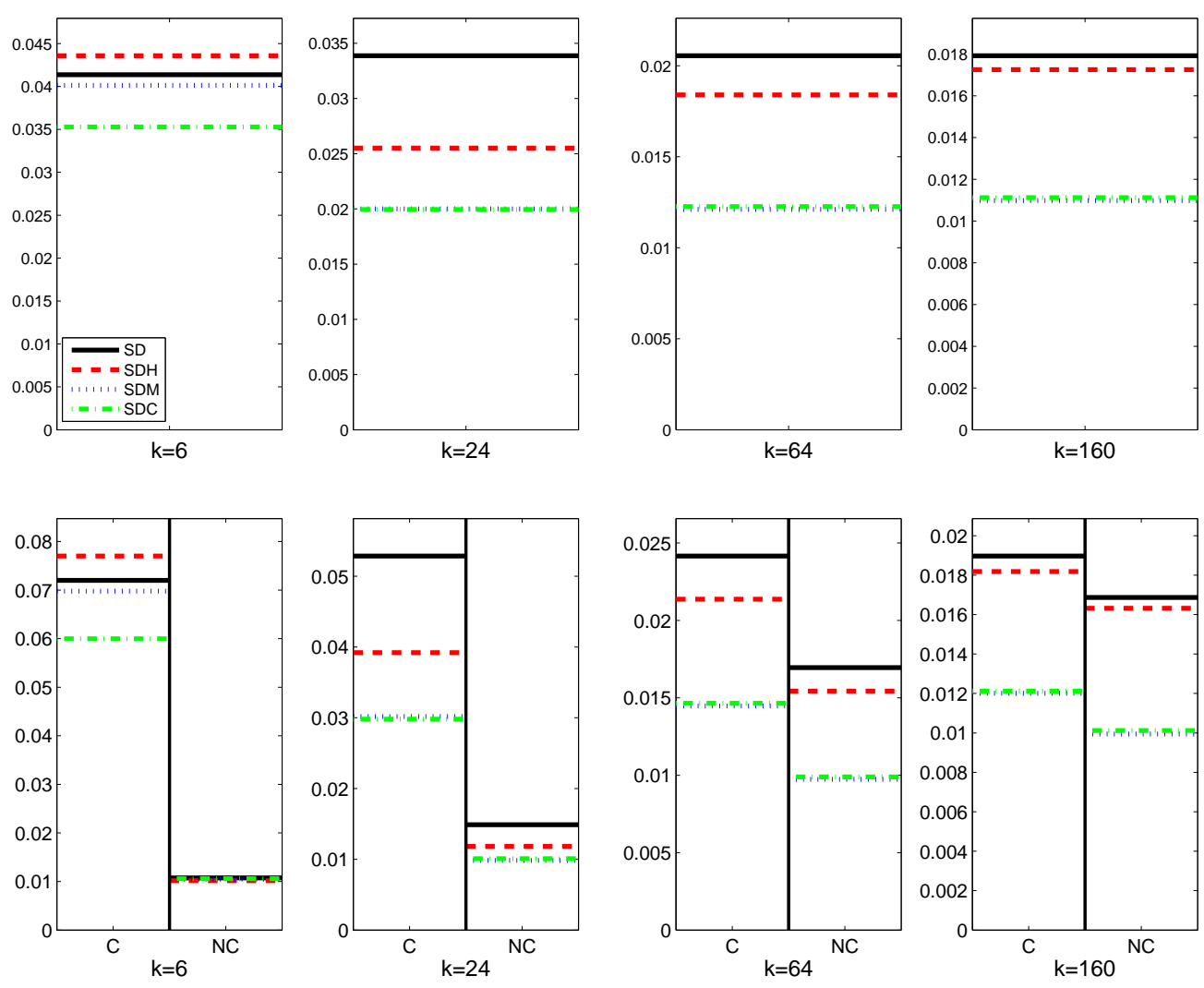

Fig. 10. Simulation results univariate outliers: mean squared errors for different $k$ values; various outlier configurations as indicated

the non-contaminated components, the gains in precision are similar as in the previous situations, but these gains are obviously negligible compared to the gain in robustness in the other components. These results indicate that the cellwise weighted SD estimates can potentially cope with much larger amounts of contamination than the original SD.

Let us now increase the dimension to $p=10$ and add $10 \%$ of independent contamination in the outlying direction $\mathbf{m}=(1,1,1,1,1,0,0,0,0,0)$. The results in Figure 10 again show that both SDC and SDM considerably improve the MSE of the location estimates, either due to a bias reduction of the contaminated components or due to a gain in precision. We see further that SDC and SDM perform similarly, except for contamination close to the regular observations (small $k$ ) where SDC outperforms SDM. Results for $10 \%$ of contamination in the outlying direction $(1,3,5,7,9,0,0,0,0,0)$ were similar to those in Figure 10 and are omitted here.

In Figure 11, the amount of independent contamination was increased to 20\%, 
$p=10, n=100,20 \%$ outliers at $k \times(1,1,1,1,1,0,0,0,0,0) ; k$ varies; top: MSE;

bottom: MSE of contaminated $(\mathrm{C})$ and non-contaminated $(\mathrm{NC})$ variables
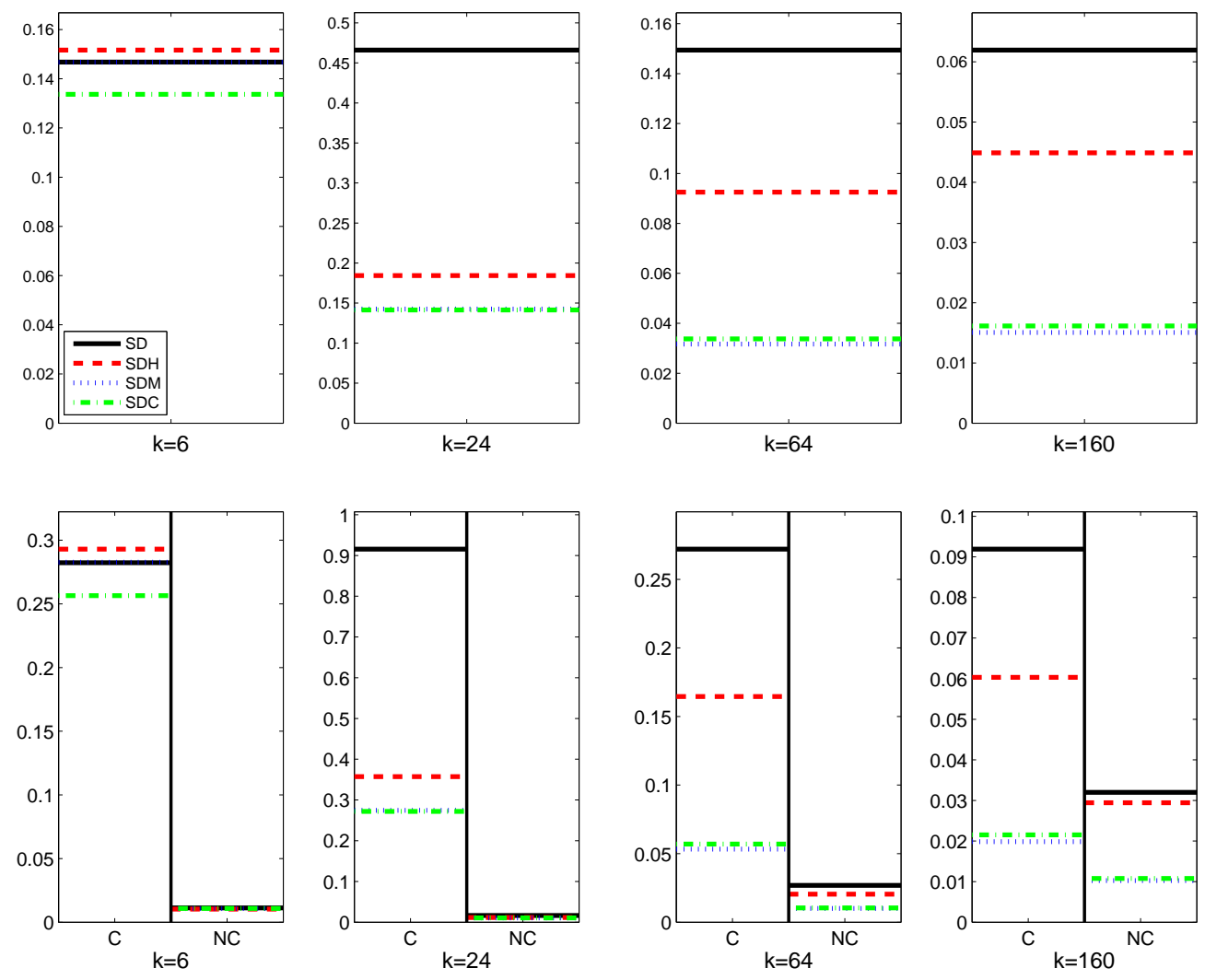

Fig. 11. Simulation results univariate outliers: mean squared errors for different $k$ values; various outlier configurations as indicated

again in direction $\mathbf{m}=(1,1,1,1,1,0,0,0,0,0)$. It can be seen that the SD location estimate has severe bias problems, which slowly improve though for increasing outlying distance $k$. For $k=6$, or contamination close to the regular observations, the cellwise weighted adaptations are not very effective at reducing the MSE. For contamination further away, however, we again observe quite a large improvement by methods SDC and SDM, mainly due to bias reduction and thus gain in robustness, but also because of gain in precision. Similar results were found for $35 \%$ of contamination.

\subsection{Structural outliers}

We also investigated the performance of the cellwise adaptations in case of correlation outliers. The main difference with (independent) componentwise outliers is that now, we have more observations which are contaminated in several components at once. Consequently, the adverse effect of bias increase 
due to observations in group (2) above is more difficult to avoid.

The correlated data were generated (similarly as in Maronna and Zamar 2002) by first drawing samples $\left\{y_{1}, \ldots, y_{n}\right\}$ of size $n=100$ in $p=10$ dimensions from a standard normal distribution and then setting $x_{i}=R y_{i}$, with

$$
R=\left(\begin{array}{cc}
A & \mathbf{0} \\
\mathbf{0} & I_{p-\tilde{p}}
\end{array}\right)
$$

where $\mathbf{0}$ represents a zero matrix and $I_{p-\tilde{p}}$ is the identity matrix of dimension $p-\tilde{p}$, with $\tilde{p}$ the number of correlated components. Matrix $A \in \mathbb{R}^{\tilde{p} \times \tilde{p}}$ is defined by

$$
A_{j j}=1 \quad \text { and } \quad A_{j k}=\rho \text { for } j \neq k
$$

Parameter $\rho$ was chosen so that the multiple correlation $\rho_{\text {mult }}$ between any coordinate of $\tilde{X}=\left(X_{1}, \ldots, X_{\tilde{p}}\right)$ and all of the others, took on chosen values. If $\rho_{\text {mult }}$ is high, then $\tilde{X}$ is concentrated around the line with direction $\mathbf{e}=$ $(1, \ldots, 1) \in \mathbb{R}^{\tilde{p}}$.

Next, correlation outliers were added as follows. For each sample, $100 \varepsilon \%$ of the data were shifted over a distance of $k \mathbf{m}$, where $\mathbf{m}$ is a unit vector. Define $\mathbf{b}$ $\in \mathbb{R}^{\tilde{p}}$ by $b_{j}=(-1)^{j}$, and set $\mathbf{a}=\mathbf{b}-\left(\mathbf{b}^{\prime} \mathbf{e} / \tilde{p}\right) \mathbf{e}$, which is orthogonal to $\mathbf{e}$. Then, the outlying direction $\tilde{\mathbf{m}}$ is chosen as $\tilde{m}_{j}=a_{j}$ (for $j \leq \tilde{p}$ ) and $\tilde{m}_{j}=0$ (for $j>\tilde{p}$ ). After normalizing $\tilde{\mathbf{m}}$ to unit norm, we obtain $\mathbf{m}$. Again the variance was reduced for the contaminated components, by multiplying their standard deviation by 0.1 . For each situation, 500 samples were generated.

Similarly as before, we consider the overall MSE as well as $\mathrm{MSE}_{C}$ and $\mathrm{MSE}_{N C}$, as defined above, where $\mathrm{MSE}_{C}$ represents the first $\tilde{p}$ components.

We first consider the case of $\rho_{\text {mult }}=0$, such that none of the components are correlated. Figure 12 presents the results for $20 \%$ of outliers and $\tilde{p}=4$. As before, the original SD suffers from some bias which diminishes as the distance $k$ increases. In the top row, we see that for small $k$, methods SDH and SDM do not succeed in reducing the MSE of the original SD estimator. In this case the higher weights of the clean observations of groups (1) and (3) above, were not sufficient to offset the bias increase due to higher weights for contaminated observations from group (2). On the other hand, method SDC overall slightly improves on the SD. For larger outlying distance $k$, as the bias decreases, the MSE becomes dominated by the variance and SDM turns out to yield the largest gain in precision in these cases, although SDC also performs well.

Results for the correlated case with $\rho_{\text {mult }}=0.9$ are shown in Figure 13. Due to the correlation, the outliers are relatively further from the regular observations than in the previous setting, and hence the bias of the original SD is limited, even for small $k$. However, the cellwise adaptations increase this bias 
$p=10, n=100, \rho_{\text {mult }}=0,20 \%$ correlation outliers at $k \times \mathbf{m}, \tilde{p}=4 ; k$ varies;

top: MSE; bottom: MSE of contaminated (C) and non-contaminated (NC) variables
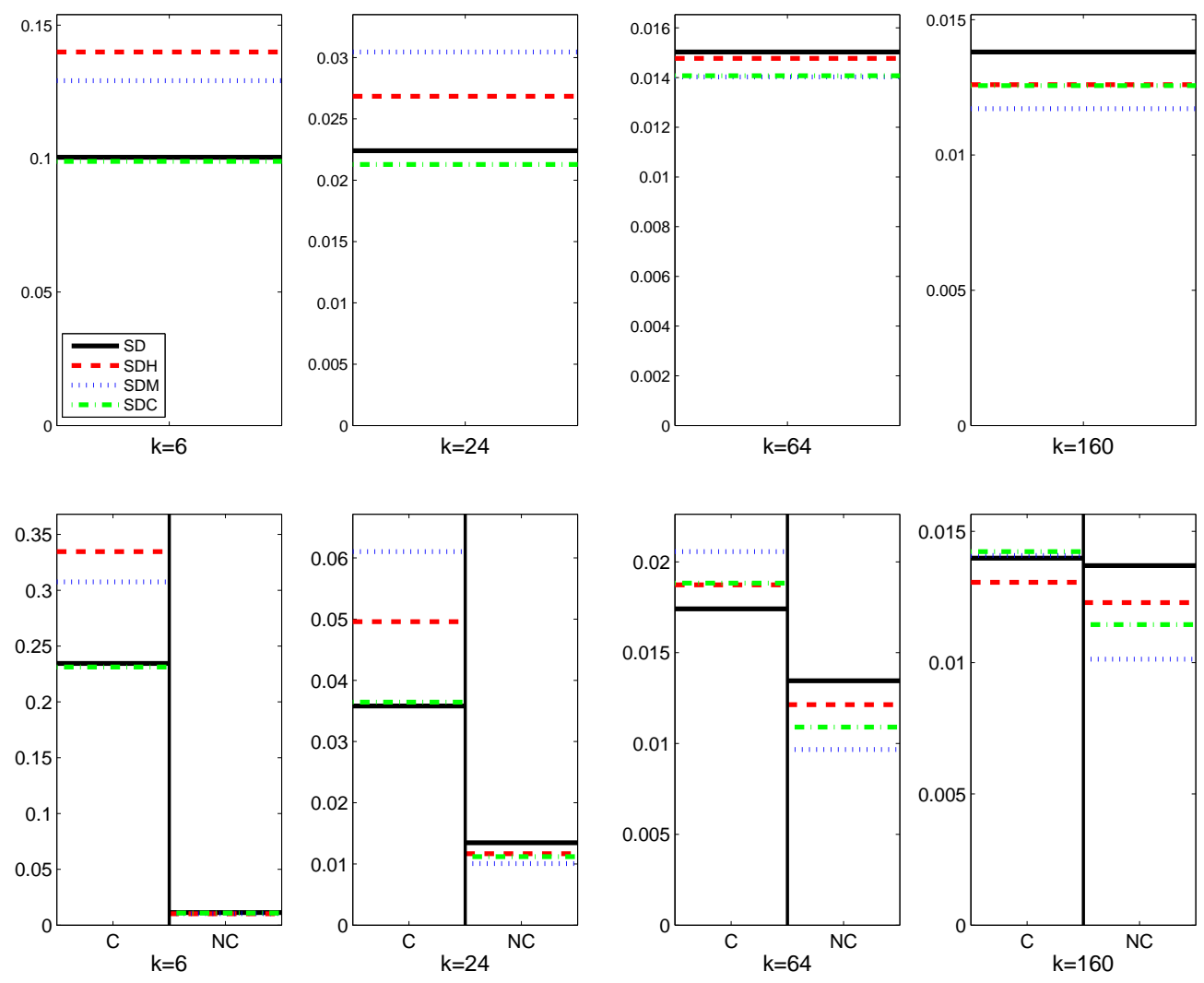

Fig. 12. Simulation results correlation outliers: mean squared errors for different $k$ values; various outlier configurations as indicated

and therefore increase the overall MSE for small $k$. Especially methods SDH and SDM perform poorly. For larger distances $k$, we again observe that SDM offers a nice gain in precision, while the other adaptations also have a positive effect on the overall MSE. Similar conclusions were found for other simulation settings involving structural outliers, which are not reported here.

We may summarize the simulation results as follows.

- In case the original SD estimates are roughly unbiased, then the cellwise adaptations have been shown to offer a relatively large reduction in MSE, corresponding to a gain in precision.

- In case the original SD estimates are not able to fully resist the contamination and suffer from bias, then

- in case of independent componentwise contamination: the cellwise adaptations SDC and SDM are quite capable of identifying the correct contaminated component(s), hence prohibiting these components from obtaining an increased weight $w_{i j}$. The bias is therefore generally not increased and 
$p=10, n=100, \rho_{\text {mult }}=0.9,20 \%$ correlation outliers at $k \times \mathbf{m}, \tilde{p}=4 ; k$ varies;

top: MSE; bottom: MSE of contaminated (C) and non-contaminated (NC) variables
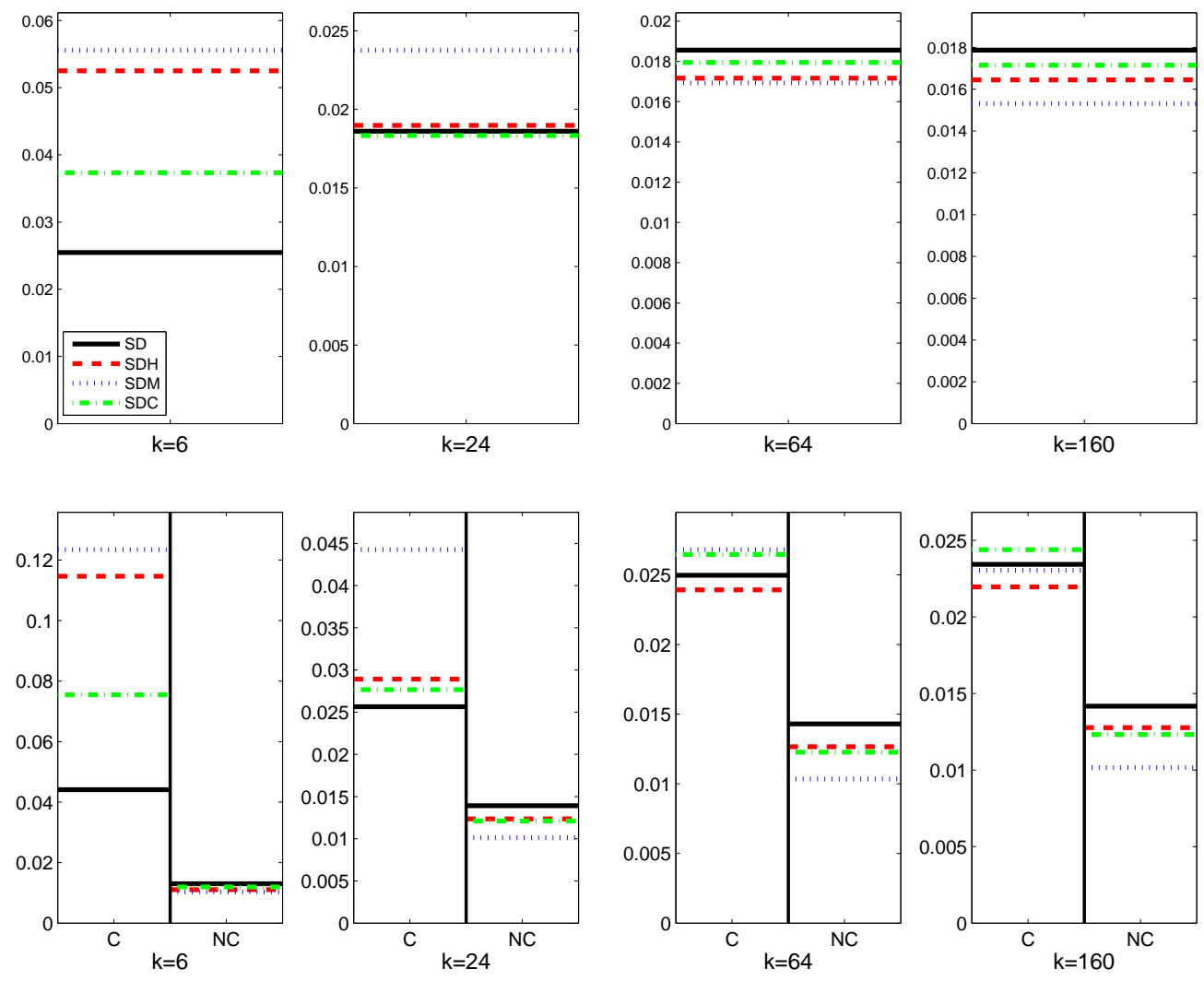

Fig. 13. Simulation results correlation outliers: mean squared errors for different $k$ values; various outlier configurations as indicated

on the contrary reduced by increased weights for many clean observations. Additionally to this gain in robustness, a gain in precision is obtained in non-contaminated components.

- in case of structural outliers: the cellwise adaptations SDC and especially SDM give evidence of difficulties identifying all of the contaminated components of an observation. Therefore some of these components may obtain a significant weight increase and this increases the bias. This loss of robustness on its turn may outweigh the gain in precision obtained in non-contaminated components.

We may conclude that the cellwise weighted adaptations in many situations offer a nice reduction of MSE with respect to the original Stahel-Donoho estimator. However, in some cases with structural outliers the methods do not perform well, with SDC being more reliable in this regard than SDM. Very positive results are obtained in the setting of independent componentwise contamination, in terms of both improving the precision and the robustness. With respect to the latter, it has been observed that both SDC and SDM can 

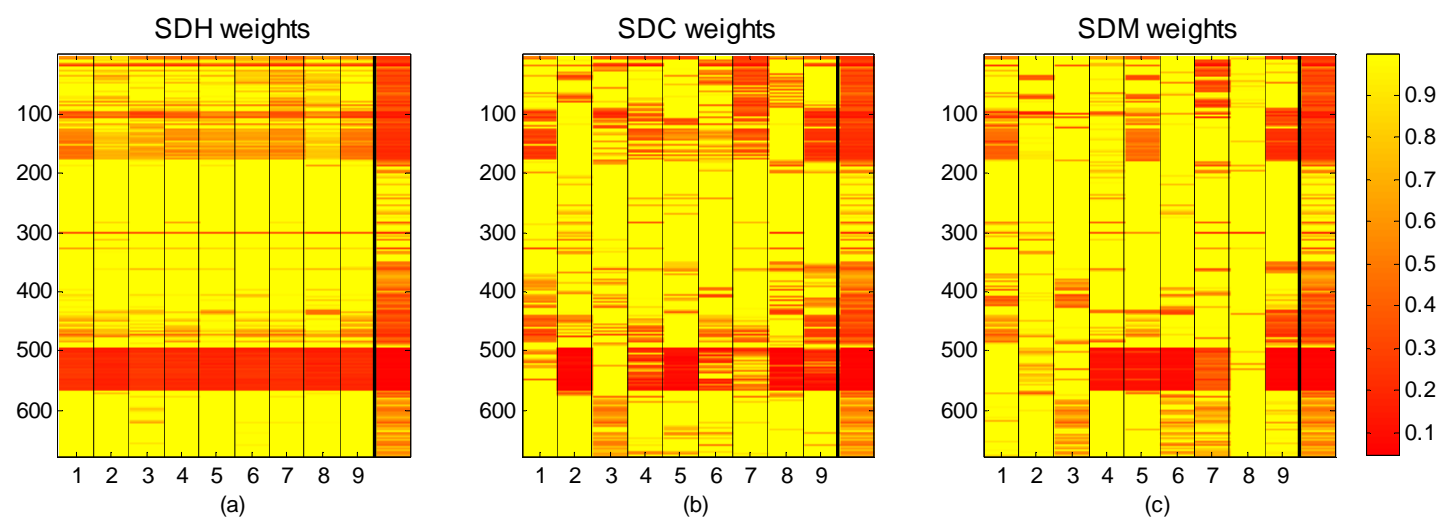

Fig. 14. Philips data: adapted SD weights for (a) SDH, (b) SDC and (c) SDM. cope with larger amounts of contamination than the SD estimate itself.

\section{$6 \quad$ Examples}

\subsection{Philips data}

Consider again the Philips data $(n=677, p=9)$, for which we have illustrated in Section 2 that the original Stahel-Donoho estimator is potentially ignoring a lot of information by assigning the same weight to each component of an outlier. Let us see now whether our adaptations of the Stahel-Donoho estimator can be useful.

Figure 14 shows the weights $w_{i j}$ for respectively SDH, SDC and SDM, in the form of heat maps. For each map, the horizontal axis represents the variable $j$ and the vertical axis the observation $i$. The colors range from dark to light, corresponding to the weights ranging from 0 to 1 . The maps have an additional column on the right, the $(p+1)$-th column, which shows the original SD weights $w_{i}$. These weights correspond to the outlyingnesses $r_{i}$ shown in Figure 1. Observations 491 to 565, for example, can again be identified as a group of strongly outlying points, based on the very low SD weights represented by the dark colors. We now take a closer look at how the componentwise adaptations deal with the outliers.

In the left plot in Figure 14 we see that the weights assigned by the SDH method to observations 491 to 565 remain low for each of the nine variables. The method takes componentwise differences into account to a limited extent only, and none of the variables is awarded a full weight repair. On the other hand, for many of the other outliers found by the SD weights, for example observations 1 to 175 , the SDH method assigns full weights to each of the variables, which is not likely to be justified since at least one component 


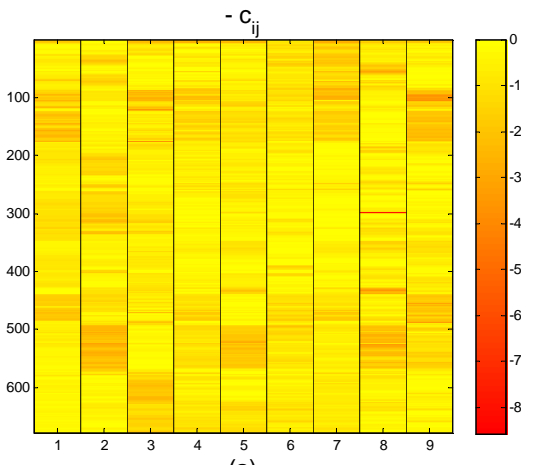

(a)

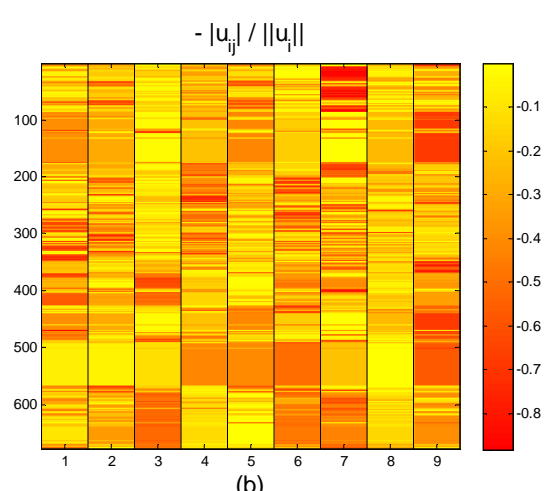

(b)

Fig. 15. Philips data: (a) (negative) componentwise outlyingnesses $c_{i j}$, (b) (negative) coefficients in maximizing directions $u_{i}$ (normalized).

should be responsible for the large outlyingness $r_{i}$. The reason for these large adapted weights is that apparently none of the componentwise outlyingnesses $c_{i j}$ is reasonably large compared to $r_{i}$ and $\mathrm{SDH}$ is not able to take into account combinations of components.

As can be seen in the middle and right plots in Figure 14, both SDC and SDM yield a much stronger distinction between the nine components than SDH did. The two methods act differently though. Concerning the group of observations 491 to 565, the SDC method mostly retains the low weight for components V2, V5 and V8, while the weights for components V1, V3 and V7 are considerably increased. On the other hand, the SDM method mainly holds components V4, V5, V6 and V9 responsible while fully repairing the weights of V1, V2, V3 and V8. Some differences between SDC and SDM can be noticed regarding the other outliers as well. All of these differences can of course directly be traced back to the behavior of respectively the componentwise outlyingnesses $c_{i j}$ and the maximizing direction coefficients $u_{i j}$. Figure $15(\mathrm{a})$ shows the values of $-c_{i j}$ while Figure 15(b) depicts $-\left|u_{i j}\right| / \| u_{i}||$. The minus was added here to arrange that large values (light colors) be comparable to large values (light colors) in the plots of $w_{i j}$ in Figure 14. We see for example that for observations 491 to 565 the value of $c_{i j}$ is indeed relatively high in components V2, V5 and V8, while components V4, V5 and especially V6 and V9 exhibit the largest coefficients in the corresponding directions $u_{i}$.

Hence, estimators $\left(T_{S D C}, S_{S D C}\right)$ and $\left(T_{S D M}, S_{S D M}\right)$ both take componentwise differences into account. The question then arises which method acts most appropriately here. Let us focus on observations 491 to 565 and recall Figure 3(b), which showed pairwise scatter plots for component V6. It could be seen that this group of points was clearly outlying with regard to the correlation between V6 and V9, as well as that between V6 and V5. Therefore we may conclude that at least components V5, V6 and V9 should retain a low weight and in this view SDM performs much better than SDC. Moreover, closer inspection suggests that the SDM results are generally supported by all pairwise 


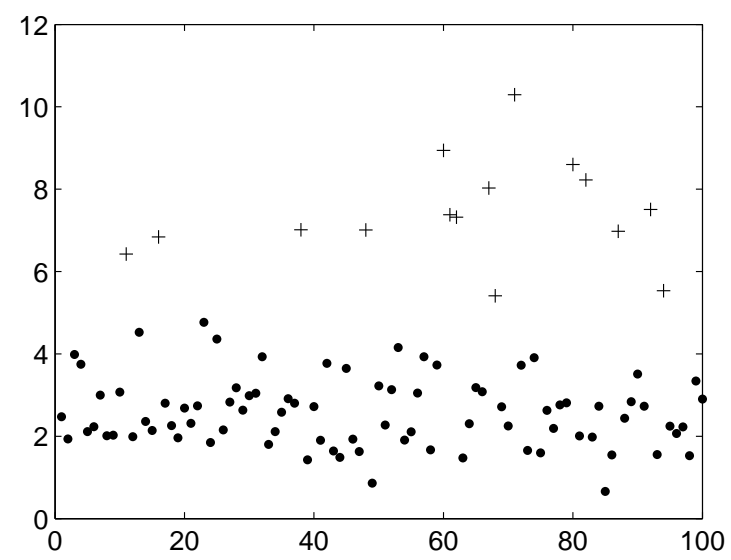

Fig. 16. Stahel-Donoho outlyingnesses of the Bank notes data.

scatter plots (not shown here). On the other hand, we have seen in Figure 2 that V2 is the component in which the outlyingness of observations 491 to 565 seems most extreme. Nevertheless, component V2 of these outliers is assigned weight 1 by the SDM method, while SDC retains a very low weight for this component. This could be seen as a shorthcoming of the SDM method, although it is open to interpretation. Indeed, one might as well argue that SDM righteously viewed the outlyingness in V2 as negligible and succeeded in identifying instead the truly important components to downweight. To conclude this example, although preceding simulation results indicated that the estimator $\left(T_{S D M}, S_{S D M}\right)$ can be inaccurate in some cases, the SDM method here seems to act appropriately and outperforms the other methods. Given the number of components that were awarded a large weight increase, the adapted $\left(T_{S D M}, S_{S D M}\right)$ estimates clearly use considerably more information than the original $\left(T_{S D}, S_{S D}\right)$ estimates and hence are likely to be more efficient.

\subsection{Bank notes data}

For our second example, we consider the Swiss bank notes data, consisting of $n=100$ forged old Swiss 1000 franc bills (Flury and Riedwyl , 1988). The variables correspond to $p=6$ different measurements, such as the length and height of the bill. The Stahel-Donoho outlyingnesses $r_{i}$ of the observations are shown in Figure 16. A set of 15 outliers is highlighted. We are now again interested in the componentwise adaptations of the weights of these outliers provided by SDH, SDC and SDM.

Figure 17 depicts the weights $w_{i j}$ for the three respective methods, with the original SD weight $w_{i}$ again represented by the additional column on the righthand side of each plot. In these plots, on the vertical axis the observations are now shown in the order of decreasing weight $w_{i}$, which aids the clarity of the 


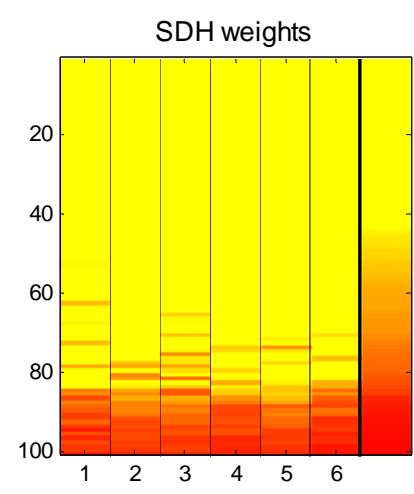

(a)

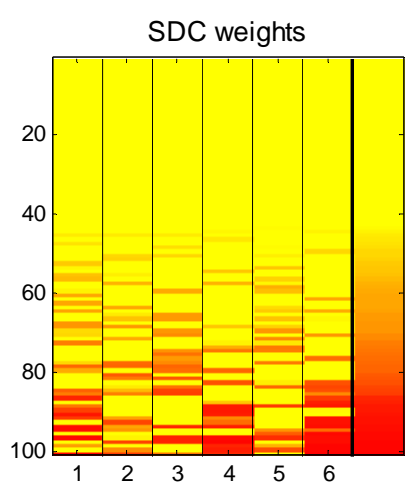

(b)

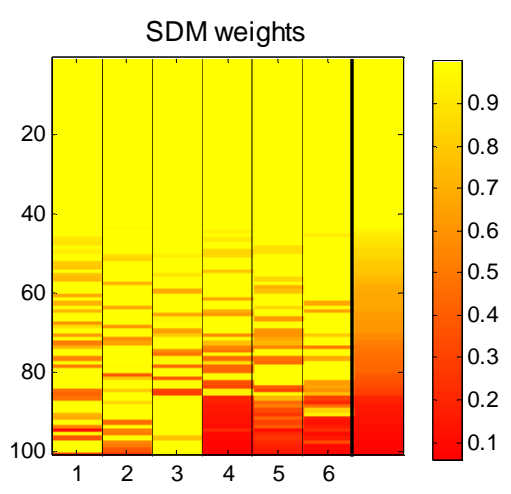

(c)

Fig. 17. Bank notes data: adapted SD weights for (a) SDH, (b) SDC and (c) SDM.

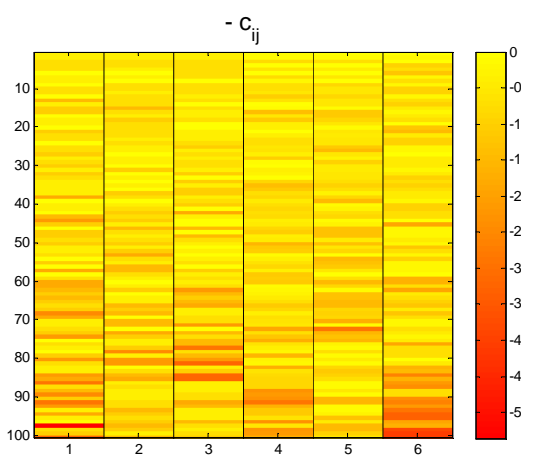

(a)

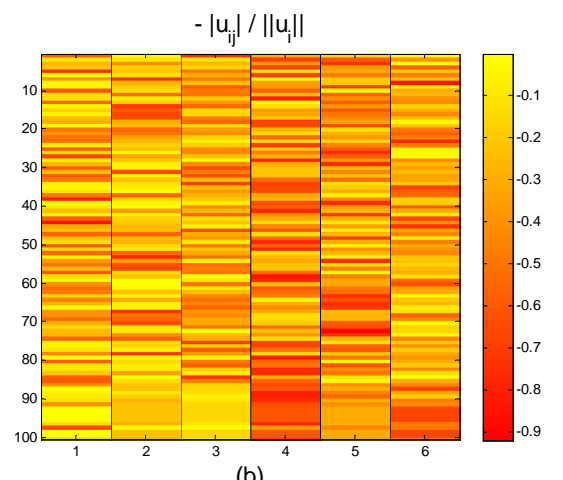

(b)

Fig. 18. Bank notes data: (a) (negative) componentwise outlyingnesses $c_{i j}$, (b) (negative) coefficients in maximizing direction $u$ (normalized).

results in this example. Figure 18 then again shows the corresponding values of $-c_{i j}$ and $-\left|u_{i j}\right| /\left\|u_{i}\right\|$ for comparison.

First, we see in the left plot of Figure 17 that the SDH weights deviate little from the original SD weights. In particular for the outlying observations, the different components are often treated equally and few components have been restored through a higher weight. The SDC (middle plot) and SDM (right plot) on the other hand, both assign very distinct weights to the various components of the outliers. As in the previous example, the two methods do not fully agree on which components to hold responsible for the large outlyingnesses. The SDC method mainly downweights components V1, V4 and V6 in accordance with the componentwise outlyingnesses $c_{i j}$ of Figure 18(a), while SDM largely targets components V4, V5 and V6 following the coefficients in the maximizing directions $u_{i}$ in Figure 18(b).

Figure 19 shows the pairwise scatter plots for all six variables in the Bank notes data. The observations identified as outliers in Figure 16 are marked in dark. First note that these points are not seriously outlying in any of the individual components. However, we see that the points clearly are correlation, or structural, outliers with regard to components V4 and V6. To a lesser extent 
the points also are deviating with regard to the correlations between $\mathrm{V} 4$ and V5 and between V1 and V6. Since both SDC and SDM have recognized the responsibility of V4 and V6, we may conclude that both methods performed satisfactorily. The methods then only differ in the way they emphasized the minor contribution of V1 rather than that of V5 or vice versa. Finally, both SDC and SDM repair the weights of about half of the components of the outliers, which is likely to constitute an appreciable gain in precision for the adapted Stahel-Donoho estimates $\left(T_{S D C}, S_{S D C}\right)$ and $\left(T_{S D M}, S_{S D M}\right)$.
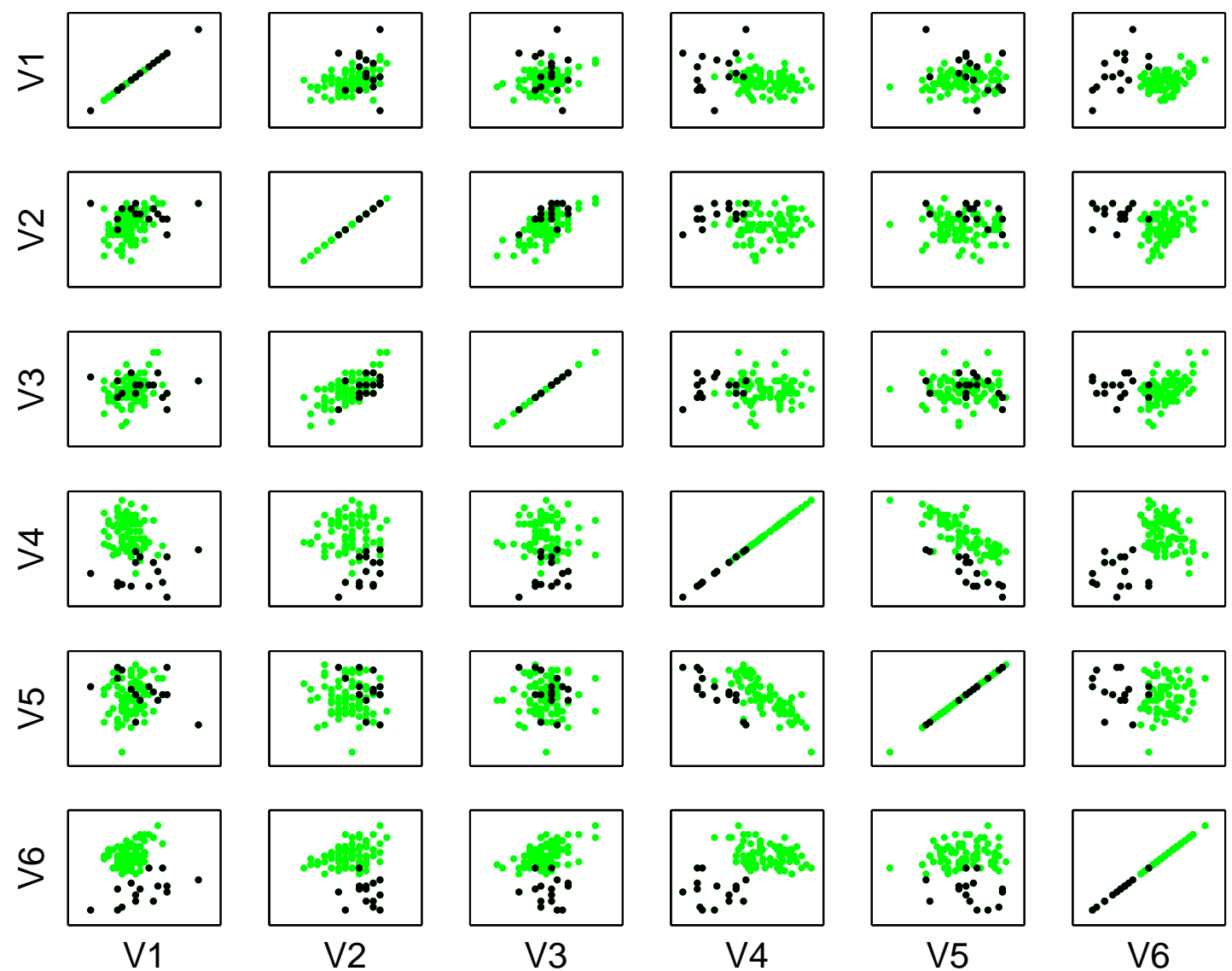

V5

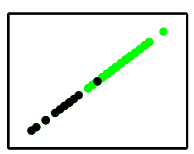

V6

Fig. 19. Bank notes data: matrix of all pairwise plots. The $(\mathrm{i}, \mathrm{j})$-th element of the matrix shows the scatter plot of the $\mathrm{i}$-th variable against the $\mathrm{j}$-th variable. The outlying observations are marked in dark.

\section{Discussion and conclusion}

We presented an adaptation of the Stahel-Donoho estimator with separate weights for each component of the observations. Three cellwise weighted adap- 
tations, denoted as $\left(T_{S D H}, S_{S D H}\right),\left(T_{S D C}, S_{S D C}\right)$ and $\left(T_{S D M}, S_{S D M}\right)$, were considered. It was shown that both SDC and SDM offer a considerable increase in weights for non-contaminated components for many outlier situations. This gain in precision may come at a cost of increased weights for contaminated components, and thus loss of robustness. But this cost is limited and a simulation study showed that in many situations the cellwise weighted adaptations enjoy a lower mean squared error than the original Stahel-Donoho estimator. Especially in the setting of independent componentwise contamination, our methods yield very good results. In some cases with structural outliers, however, the gain in precision does not outweigh the loss of robustness. It is clear that further research is required to optimize the cellwise weighting method, in particular to avoid the loss of robustness as much as possible. The results in this paper are promising and are meant to show that research in this direction is worthwhile. Finally, apart from increasing the precision of the location and scatter estimates, the identification of contaminated components in outliers may be of interest in its own right in the context of outlier detection.

\section{References}

Alqallaf, F., Van Aelst, S., Yohai, V.J., Zamar, R.H. (2007). Propagation of Outliers in Multivariate Data. Annals of Statistics, to appear.

Debruyne, M., Hubert, M. (2008). The influence function of the Stahel-Donoho covariance estimator of smallest outlyingness. Statistics and Probability Letters, to appear.

Donoho, D. L., 1982. Breakdown Properties of Multivariate Location Estimators. Ph.D. qualifying paper, Harvard University.

Flury, B., Riedwyl, H., 1988. Multivariate Statistics. A practical Approach, Chapman and Hall, London.

Gervini, D., 2002. The influence function of the Stahel-Donoho estimator of multivariate location and scatter. Statistics and Probability Letters, Vol. 60, 425-435.

Huber, P.J., 1964. Robust estimation of a location parameter. The Annals of Mathematical Statistics, Vol. 35, 73-101.

Hubert, M., Rousseeuw, P.J., Vanden Branden, K. (2005). ROBPCA: a new approach to robust principal component analysis. Technometrics, Vol. 47, $64-79$.

Hubert, M., Verboven, S. (2003). A robust PCR method for high-dimensional regressors. Journal of Chemometrics, Vol. 17, 438-452.

Maronna, R. A., Yohai, V. J., 1995. The behavior of the Stahel-Donoho Robust Multivariate Estimator. Journal of the American Statistical Association, Vol. 90, No. 429, $329-341$.

Gather, U., Hilker, T., 1997. A Note on Tyler's Modification of the MAD for the Stahel-Donoho Estimator. The Annals of Statistics, Vol. 25, No. 5, 
$2024-2026$.

Rousseeuw, P. J., Van Driessen, K., 1999. A fast algorithm for the Minimum Covariance Determinant Estimator. Technometrics, Vol. 41, No. 3, 212-223. Stahel, W. A., 1981. Breakdown of Covariance Estimators. Research Report 31, Fachgruppe für Statistik, E.T.H. Zürich.

Tukey, J.W., 1962. The Future of Data Analysis. The Annals of Mathematical Statistics, Vol. 33, 1-67.

Zuo, Y., Cui, H., He, X., 2004. On the Stahel-Donoho estimator and depthweighted means of multivariate data. Annals of Statistics, Vol. 32, $167-188$. 